\title{
IncRNA DRAIR is downregulated in diabetic monocytes and modulates the inflammatory phenotype via epigenetic mechanisms
}

\author{
Marpadga A. Reddy, ${ }^{1}$ Vishnu Amaram, ${ }^{1}$ Sadhan Das, ${ }^{1,2}$ Vinay Singh Tanwar, ${ }^{1}$ Rituparna Ganguly, ${ }^{1}$ \\ Mei Wang, ${ }^{1}$ Linda Lanting, ${ }^{1}$ Lingxiao Zhang, ${ }^{1}$ Maryam Abdollahi, ${ }^{1}$ Zhuo Chen, ${ }^{1}$ Xiwei $\mathbf{W u}{ }^{3}$ \\ Sridevi Devaraj, ${ }^{4}$ and Rama Natarajan ${ }^{1}$ \\ 'Department of Diabetes Complications and Metabolism, Arthur Riggs Diabetes and Metabolism Research Institute \\ and Beckman Research Institute of City of Hope, Duarte, California, USA. ${ }^{2}$ Division of Pharmacology, CSIR-Central Drug \\ Research Institute, Lucknow, India. ${ }^{3}$ Integrative Genomics Core, Beckman Research Institute of City of Hope, Duarte, \\ California, USA. ${ }^{4}$ Baylor College of Medicine and Texas Children's Hospital, Houston, Texas, USA.
}

\begin{abstract}
Long noncoding RNAs (IncRNAs) are increasingly implicated in the pathology of diabetic complications. Here, we examined the role of IncRNAs in monocyte dysfunction and inflammation associated with human type 2 diabetes mellitus (T2D). RNA sequencing analysis of CD14 monocytes from patients with $\mathrm{T} 2 \mathrm{D}$ versus healthy controls revealed downregulation of antiinflammatory and antiproliferative genes, along with several IncRNAs, including a potentially novel divergent IncRNA diabetes regulated antiinflammatory RNA (DRAIR) and its nearby gene CPEB2. High glucose and palmitic acid downregulated DRAIR in cultured CD14+ monocytes, whereas antiinflammatory cytokines and monocyte-to-macrophage differentiation upregulated DRAIR via KLF4 transcription factor. DRAIR overexpression increased antiinflammatory and macrophage differentiation genes but inhibited proinflammatory genes. Conversely, DRAIR knockdown attenuated antiinflammatory genes, promoted inflammatory responses, and inhibited phagocytosis. DRAIR regulated target gene expression through interaction with chromatin, as well as inhibition of the repressive epigenetic mark $\mathrm{H3K} 9 \mathrm{me2}$ and its corresponding methyltransferase C9a. Mouse orthologous Drair and Cpeb2 were also downregulated in peritoneal macrophages from $\mathrm{T} 2 \mathrm{D} d \mathrm{~b} / \mathrm{db}$ mice, and Drair knockdown in nondiabetic mice enhanced proinflammatory genes in macrophages. Thus, DRAIR modulates the inflammatory phenotype of monocytes/macrophages via epigenetic mechanisms, and its downregulation in T2D may promote chronic inflammation. Augmentation of endogenous IncRNAs like DRAIR could serve as novel antiinflammatory therapies for diabetic complications.
\end{abstract}

Conflict of interest: The authors have declared that no conflict of interest exists.

Copyright: (c) 2021, Reddy et al. This is an open access article published under the terms of the Creative Commons Attribution 4.0 International License.

Submitted: August 17, 2020

Accepted: April 28, 2021

Published: June 8, 2021

Reference information: /CI Insight. 2021;6(11):e143289.

https://doi.org/10.1172/jici. insight.143289.

\section{Introduction}

Systemic low-grade inflammation is a hallmark of type 2 diabetes mellitus (T2D) and contributes to the pathogenesis of several associated complications, including cardiovascular disease $(1,2)$. Activation of monocytes and macrophages plays an important role in inflammatory processes needed for protection against invading pathogens or toxins (3). Under physiological conditions, acute inflammation self-resolves via a balancing interplay between inflammatory and antiinflammatory mediators and is essential for tissue repair $(4,5)$. However, factors associated with inflammatory diseases like T2D, including high glucose (HG), elevated levels of advanced glycation end products (AGEs), and free fatty acids (e.g., palmitic acid [PA] and oxidized lipids), markedly enhance production of proinflammatory cytokines such as IL-1 $\beta$, IL-6, and TNF- $\alpha$ and inhibit production of protective factors, leading to chronic inflammation. In addition, the diabetic milieu increases the number of monocytes (monocytosis), further increasing the burden for cardiovascular disease (5-10). Experimental studies have elucidated key molecular and signaling pathways involved in the activation of monocytes and macrophages. These include increased oxidative stress; production of ROS; and activation of various kinases, transcription factors (TFs) (e.g., NF-kB and STAT3), 
inflammasomes, and TLRs (5-7). Furthermore, epigenetic mechanisms have also been demonstrated in the activation of the proinflammatory phenotype of monocytes and macrophages (11). Evidence shows that blocking inflammatory signaling using antibodies against cytokines, cytokine receptor antagonists, lipid lowering drugs, and some antioxidants could reduce risk for metabolic disease and vascular complications (12-15). However, these therapeutic strategies are not always fully effective in preventing progression and recurrence of cardiometabolic disease. Thus, further understanding of the precise molecular mechanisms associated with chronic inflammation is needed to develop much-needed new and effective therapies.

Noncoding RNAs (ncRNAs) like microRNAs (miRNAs) and long noncoding RNAs (lncRNAs) have emerged as key regulators of gene expression mediating functions of monocytes and macrophages such as inflammation, innate immunity, differential response to endotoxemia, cholesterol homeostasis, and macrophage polarization $(16,17)$. IncRNAs are defined as $>200$ nucleotide-long transcripts with no coding potential. They regulate gene transcription via diverse mechanisms depending on their subcellular localization. Nuclear lncRNAs can regulate gene expression by interacting with chromatin, or they can act as a scaffolds, decoys, or recruiters of chromatin modifying factors/complexes and TFs to alter epigenetic states at target gene promoters and enhancers. On the other hand, cytoplasmic lncRNAs can regulate functions of translation factors, signaling proteins, and miRNAs $(16,17)$.

Emerging evidence shows that lncRNAs can play functional roles in diabetic vascular disease (1820). Our recent studies demonstrated the involvement of 3 lncRNAs in the regulation of the monocyte/macrophage inflammatory phenotype in diabetes and metabolic syndrome in mice and humans. Two of these lncRNAs, E330013P06 and Dnm3os, were upregulated in macrophages from T2D $d b / d b$ mice and T2D humans relative to healthy nondiabetic controls, and these lncRNAs had proinflammatory properties $(21,22)$. In contrast, another lncRNA, Mist, was downregulated in macrophages from high-fat diet-induced obese mice, as well as in adipose tissue macrophages from humans with obesity and metabolic syndrome, and exhibited antiinflammatory properties (23). Further mechanistic studies demonstrated that disruption of lncRNA-regulated epigenetic mechanisms under diabetic or obese conditions can facilitate proinflammatory phenotypes in macrophages $(22,23)$. Several 1 ncRNAs dysregulated in cardiometabolic disease were also identified in human monocytes (24). However, very little is known about lncRNAs in human monocytes with protective effects that might assist in resolution of inflammation in T2D. Such knowledge could assist in the development of newer therapies for diabetes and its associated chronic inflammatory disorders.

In this study, we compared the transcriptome in $\mathrm{CD} 14^{+}$monocytes from $\mathrm{T} 2 \mathrm{D}$ volunteers versus monocytes from nondiabetic volunteers to gain insights into the role of differentially expressed lncRNAs in monocyte dysfunction. We found that not only many inflammatory genes were upregulated, but several antiinflammatory and antiproliferative genes were downregulated; furthermore, several lncRNAs were dysregulated in T2D monocytes versus controls. Among the differentially expressed IncRNAs, we selected 1 potentially novel 1 ncRNA for further characterization, which we named diabetes regulated antiinflammatory IncRNA (DRAIR). DRAIR expression was downregulated in T2D monocytes and in cultured human monocytes treated with HG and PA, but it was upregulated by antiinflammatory cytokines IL-4 and IL-13. Functional and mechanistic studies demonstrated that DRAIR increases the expression of antiinflammatory genes via interaction with chromatin and modulation of repressive epigenetic histone modifications at target gene promoters. Furthermore, the antiinflammatory function of Drair (mouse ortholog) was also observed in vivo in mice. Together, these studies demonstrate that lncRNA $D R A I R$ regulates the antiinflammatory phenotype via epigenetic mechanisms in monocytes and that its downregulation in diabetes promotes chronic inflammation.

\section{Results}

$T 2 D$ is associated with reduced expression of antiinflammatory and antiproliferation genes in human CD14+ monocytes. Although evidence shows that T2D promotes monocyte activation and monocytosis associated with chronic inflammation, the dysregulated gene expression and the regulatory role of 1 ncRNAs in these inflammatory processes are unclear. To examine this further, we performed strand-specific RNA sequencing (RNAseq) analysis to profile changes in the transcriptome of $\mathrm{CD}_{1} 4^{+}$monocytes obtained from human volunteers with T2D (T2D monocytes) relative to control healthy volunteers without diabetes (control monocytes) (Figure 1A and Supplemental Table 1; supplemental material available online with this article; https:// doi.org/10.1172/jci.insight.143289DS1). T2D monocytes exhibited extensively altered transcriptomes with 
upregulation of 993 genes and downregulation of 1865 genes (fold-change $\geq 2$, average coverage $\geq 1$, FDR $\leq 0.05, n=5$ per group) versus control monocytes (Figure 1B). Several inflammatory genes were upregulated (Supplemental Figure 1A), in line with previous studies (25-27). Interestingly, there was much greater reduction in the expression of several key antiinflammatory, antioxidant, and antiproliferative genes, including $I L 1 R N$ that codes for IL-1 receptor antagonist (IL-1Ra) and SOD2 and PTEN - in T2D monocytes, respectively (Figure 1C) - suggesting that T2D is associated with the loss of protective genes.

Gene ontology (GO) analysis showed enrichment of processes associated with translation, wound healing, ncRNA processing, and immune cell activation in the upregulated genes. Downregulated genes showed enrichment of intracellular signaling, immune response, and apoptosis (Supplemental Figure 1, B and C). Ingenuity Pathway Analysis (IPA) revealed enrichment of inflammatory response under the Diseases and Functions category in both the upregulated and downregulated genes (Supplemental Figure 1, D and E). Moreover, IPA also revealed enrichment of canonical pathways related to fatty acid oxidation and nitric oxide (NO) signaling among upregulated genes (Supplemental Figure 1F). Downregulated genes were enriched with several overlapping networks including NF- $\mathrm{BB}$ signaling, $\mathrm{PI} 3 \mathrm{~K} / \mathrm{Akt}$ activation, nitric oxide (NO)/ROS production, and the IL-6 pathway (Figure 1D and Supplemental Figure 1G). Together, these data suggest that disruption of protective mechanisms/factors may activate inflammatory pathways in T2D. Motif analysis revealed that promoters of differentially expressed genes (DEGs) were enriched in binding sites for key TFs such as NF- $\mathrm{B}$, Egr1, and KLF4 (Figure 1, E and F), which are known to be involved in inflammation and macrophage polarization (28). These results suggest that T2D-induced changes in the monocyte transcriptome can dysregulate genes associated with key monocyte/macrophage functions - including inflammatory phenotype, apoptosis, proliferation, and phagocytosis — that can contribute to inflammatory diabetes complications, including cardiovascular disease.

Dysregulation of IncRNAs in T2D monocytes, including a potentially novel $\operatorname{lncRNA} D R A I R$. To elucidate the role of 1 ncRNAs in T2D-induced monocyte dysfunction, we further analyzed differentially expressed lncRNAs by assessing potential open reading frames, as described (21). We found that $335 \operatorname{lncRNAs}$ were differentially expressed in T2D monocytes (Figure 2A), including those expressed from bidirectional promoters and designated as divergent transcripts (Figure $2 \mathrm{~B})$. GO analyses of nearby ( $\pm 250 \mathrm{~kb}$ ) DEGs revealed enrichment of inflammation and immune cell functions near downregulated lncRNAs (Supplemental Figure 2A), while metabolic processes were enriched in DEGs near upregulated lncRNAs (Supplemental Figure 2B).

Next, we tested our hypothesis that key lncRNAs downregulated in T2D may alter the inflammatory phenotype of monocytes and macrophages. We focused on a differentially expressed lncRNA annotated as $C P E B 2-A S$ (hg19), whose regulation and function have not been previously studied. CPEB2-AS is divergently transcribed adjacent to the cytoplasmic element binding protein 2 (CPEB2) gene on human chromosome 4. Both share the same promoter as evidenced by H3K4me3 enrichment (Figure 2C). Divergent transcripts have been reported in development (29), but their function in T2D-induced inflammation is unknown. Moreover, the nearby $C P E B 2$ belongs to the CPEB family, which has reported antiinflammatory functions (30), suggesting that $C P E B 2-A S$ may regulate inflammatory pathways. Based on subsequent functional assessment studies, we renamed CPEB2-AS as DRAIR.

RNA-seq data revealed that the $D R A I R$ and nearby $C P E B 2$ were significantly downregulated in monocytes from T2D patients (Figure 2C). Using reverse transcription followed by quantitative PCR (qPCR), we validated the significant downregulation of DRAIR and CPEB2 in CD14+ monocytes from T2D patients versus controls (Figure 2, D and E). T2D is associated with elevated blood glucose and circulating levels of free fatty acids such as PA. Therefore, we examined the effect of $\mathrm{HG}$ and PA in $\mathrm{CD} 14^{+}$monocytes from healthy volunteers in vitro. Monocytes were cultured in normal glucose (NG, $5.5 \mathrm{mM})$ or $\mathrm{HG}(25 \mathrm{mM})$ for 3 days. In the last 24 hours, NG and HG cells were also treated with PA $(200 \mu \mathrm{M})$, referred to as PA and HG + PA (HP) groups, respectively. qPCR analysis showed that - relative to NG - PA, HG, or HP significantly inhibited DRAIR expression (Figure 2F). PA also downregulated DRAIR in $\mathrm{CD} 14^{+}$monocytes converted to macrophages (Figure $2 \mathrm{G}$ ). Furthermore, HG, PA, and an inflammatory cytokine IL-1 $\beta$ (10 ng/mL, 24 hours), whose levels are elevated in T2D (2) also downregulated DRAIR expression in the human THP1 monocytic cell line (Figure 2H). These results demonstrate that lncRNA DRAIR is downregulated in T2D and by major pathological factors elevated in $\mathrm{T} 2 \mathrm{D}$, suggesting that it may regulate antiinflammatory processes in monocytes.

Characterization of DRAIR and its subcellular localization. Bioinformatics analysis using PhyloCSF and Coding Potential Calculator 2 suggested that DRAIR lacks coding potential (Supplemental Figure 3A). 
A
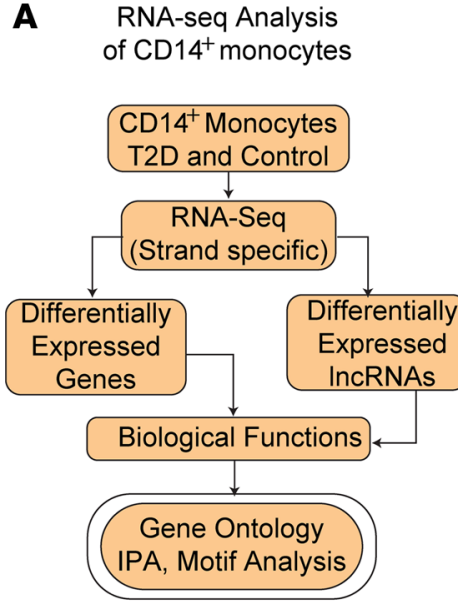

B

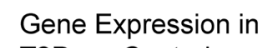

T2D vs. Controls

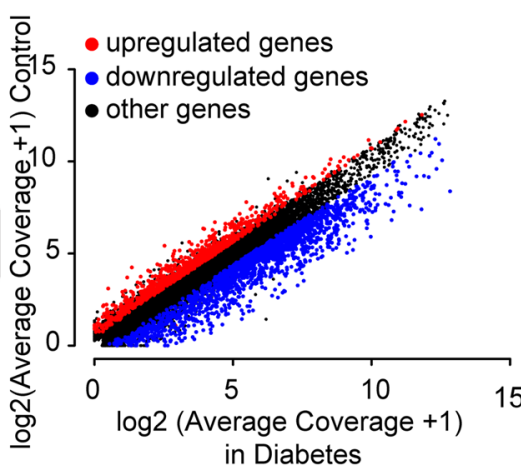

C Downregulated genes in T2D

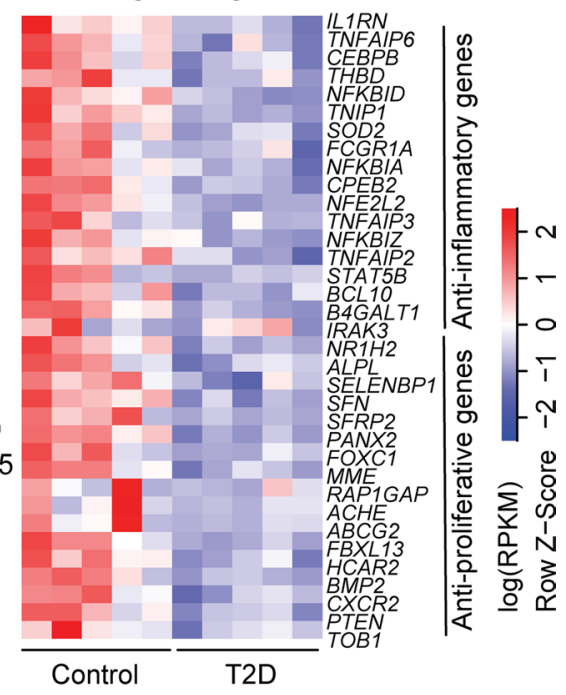

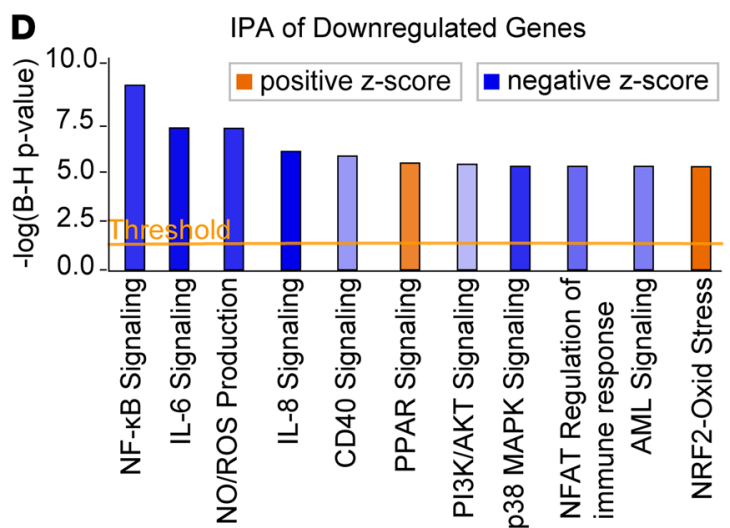

E Upregulated Genes

F Downregulated Genes

\begin{tabular}{|c|c|c|c|c|c|}
\hline Motif & TF & P-value & Motif & TF & P-value \\
\hline CCUfग & ELK1 & $2.44 e-50$ & $\operatorname{CCle~CCoce~}$ & SP1 & $1.01 \mathrm{e}-123$ \\
\hline W & TFAP2A & $4.34 \mathrm{e}-43$ & fll & TFAP2A & 2.08e-117 \\
\hline 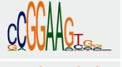 & GABPA & $1.06 e-41$ & 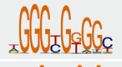 & KLF4 & $3.54 \mathrm{e}-98$ \\
\hline 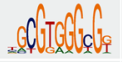 & Egr1 & $1.67 e-39$ & atvilial & EGR1 & $9.85 e-93$ \\
\hline Cafcudusc & Mycn & $1.88 e-35$ & 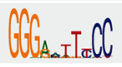 & NFKB1 & $4.73 e-68$ \\
\hline
\end{tabular}

Figure 1. Type 2 diabetes inhibits antiinflammatory and antiproliferation genes in human CD14 ${ }^{+}$monocytes. (A) Scheme showing RNA-seq analysis pipeline and downstream analyses of CD14+ monocytes from volunteers with and without T2D. (B) Scatter plot of differentially expressed genes (DEGs) in T2D monocytes versus controls ( $\log _{2}$ fold change $\geq 2$, FDR $<0.05$ versus control monocytes, $n=5$ each). (C) Heatmap showing downregulation of key antiinflammatory and antiproliferative genes in T2D monocytes. (D) Enrichment of canonical signaling pathways associated with inflammation in downregulated genes. B-H, Benjamini-Hochberg. (E and F) Transcription factor (TF) motifs enriched in the promoters (-1000 bp to +500 bp) of DEGs in T2D monocytes. Adjusted $P$ values (B-H method) are shown.

This was further confirmed experimentally in an in vitro-coupled transcription translation system using DRAIR cDNA cloned into the pcDNA3.1 expression plasmid as a template that showed absence of protein products (Supplemental Figure 3, B and D) similar to no template control (NTC) reactions. The positive control luciferase (LUC) transcript expressed a $62 \mathrm{kDa}$ protein, as expected.

Because lncRNA functions are dependent upon subcellular localization, we next examined DRAIR levels in the cytoplasmic and nuclear fractions isolated from THP1 monocytes and THP1 cells differentiated into macrophages using phorbol myristate acetate (PMA, $20 \mathrm{ng} / \mathrm{mL}$ ). qPCR showed that DRAIR levels were highly enriched in nuclear fractions from THP1 monocytes and macrophages. As expected, the known nuclear lncRNA NEAT1 and coding RNA PPIA showed enrichment in nuclear and cytosolic fractions, respectively (Supplemental Figure 4, A-F). Nuclear localization of DRAIR was further confirmed by RNA-fluorescence in situ hybridization assays in THP1 macrophages using fluorescently labeled DRAIR probes (Supplemental Figure 4G). Furthermore, DRAIR was also found to be enriched in chromatin fractions versus soluble nuclear extracts similar to NEAT1, a known chromatin-associated lncRNA (Supplemental Figure 4, $\mathrm{H}$ and I), suggesting nuclear localized DRAIR might have functions related to transcriptional regulation in monocyte/macrophages.

DRAIR downregulates the inflammatory phenotype in monocytes and macrophages. We next examined the effect of $D R A I R$ on monocyte gene expression and the inflammatory phenotype using both gain-of-function (overexpression) and loss-of-function (gene silencing) approaches. For overexpression experiments, 


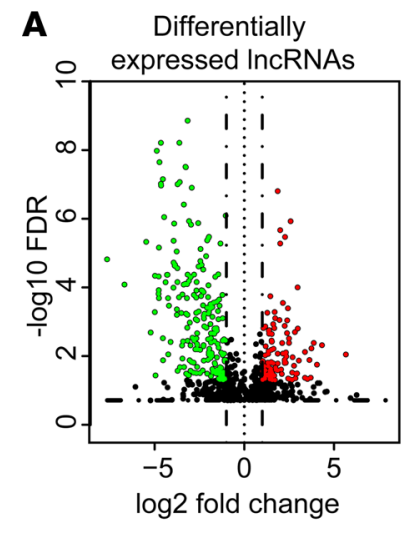

B Upregulated
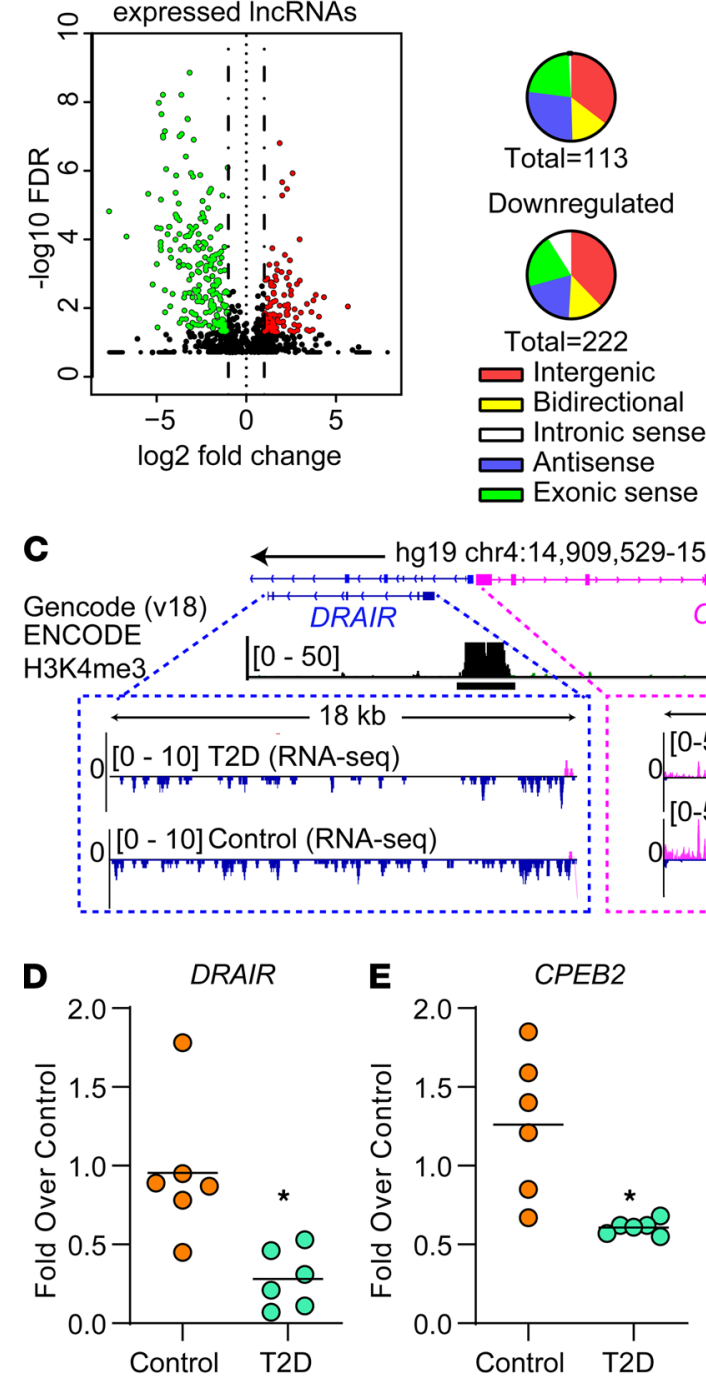

hg19 chr4:14,909,529-15,0
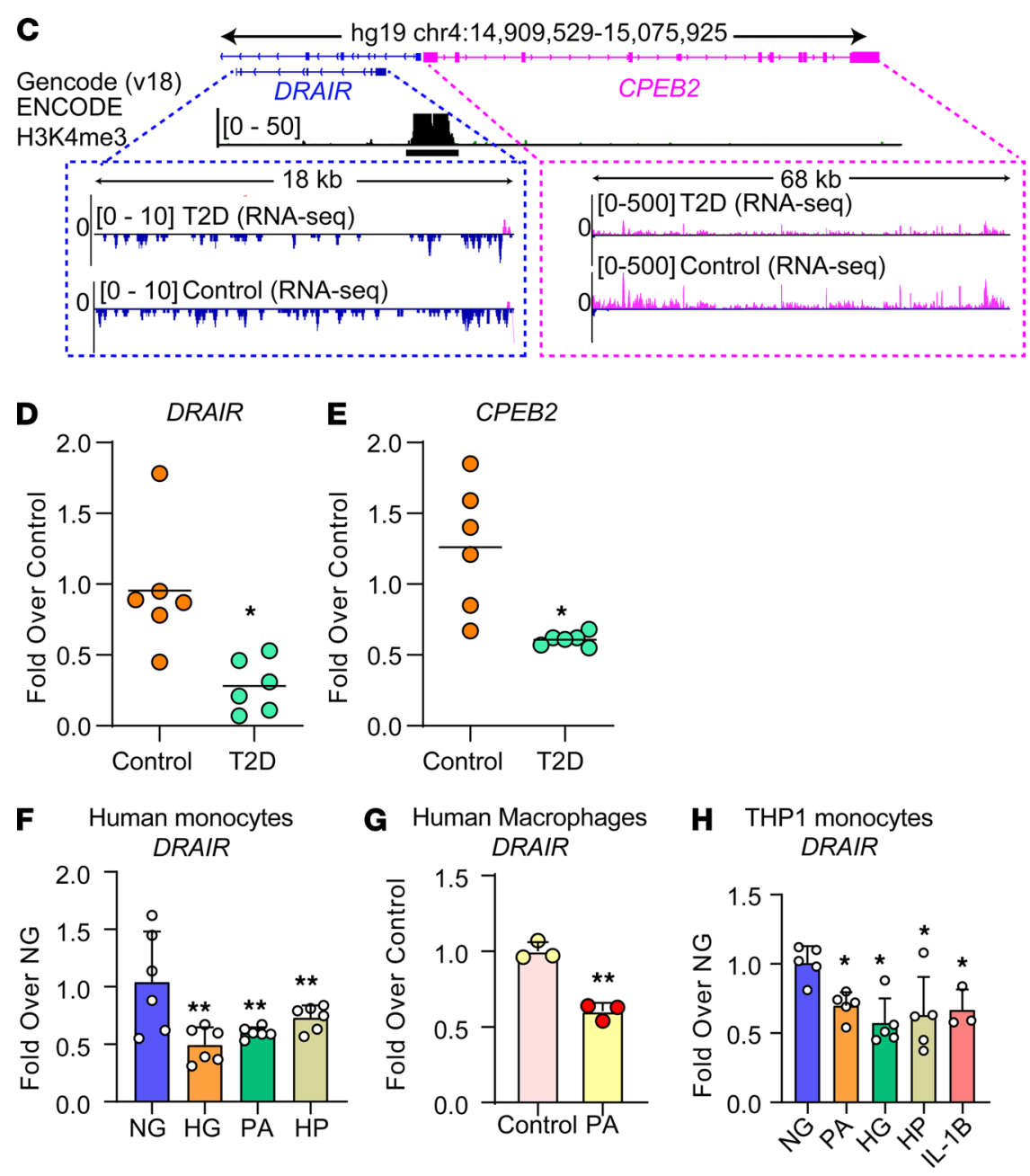

Figure 2. Dysregulation of IncRNA DRAIR in type 2 diabetes. (A and B) Volcano plot of differentially expressed IncRNAs in T2D monocytes versus controls (A) and their genomic classification (B). (C) Schematic showing genomic organization of IncRNA DRAIR and nearby CPEB2 gene, along with RNA-seq tracks from CD14+ monocytes from T2D and control subjects. DRAIR and CPEB2 show downregulation in T2D monocytes versus controls $\left(\log _{2}\right.$ fold $=-1.75$, FDR $=0.029$, and $\log _{2}$ fold $=-2.19, F D R=1.18 \times 10^{-5}$, respectively; $n=5$ each). H3K4me3 track from CD14+ monocytes (from ENCODE) is shown to indicate shared promoter region for both genes. Map not drawn to scale. ( $D$ and $\mathbf{E}$ ) qPCR validation of DRAIR and CPEB2 downregulation in T2D monocytes versus control ( ${ }^{*} P<0.05$, by $t$ tests, $\left.n=6\right)$. ( $(\mathbf{F}-\mathbf{H})$ qPCR results showing downregulation of DRAIR in primary human $C D 14^{+}$monocytes $(\mathbf{F})$, primary human macrophages (C), and THP1 monocytes (H) treated with normal glucose (NG, $5.5 \mathrm{mM}$ ), high glucose (HG, $25 \mathrm{mM}, 72$ hours), palmitic acid (PA, $200 \mu \mathrm{M}, 24$ hours), and $\mathrm{HG}+\mathrm{PA}(\mathrm{HP})$ and $\mathrm{IL}-1 \beta(10 \mathrm{ng} / \mathrm{mL}) .{ }^{*} P<0.05$ and ${ }^{*} P<0.01$, as determined by 1 -way ANOVA and Dunnett's multiple-comparison test in $\mathbf{F}$ and $\mathbf{H}(n=3-6)$ and $t$ test in $\mathbf{G}(n=3)$. 
we cloned DRAIR cDNA into a lentiviral vector. Then, we transduced THP1 monocytes with lentiviruses expressing DRAIR or a control EGFP, and the gene expression was analyzed by qPCR. Results showed that $D R A I R$ overexpression (Figure $3 \mathrm{~A}$ ) upregulated the nearby $C P E B 2$ gene and the antiinflammatory gene $I L 1 R N$ (Figure 3, B and C), but it downregulated proinflammatory genes TNF and FCGR3B (CD16b) (Figure 3, D and E). Furthermore, DRAIR overexpression also upregulated macrophage markers such as scavenger receptors $C D 68$ and $C D 36$, as well as a BCL2 family member MCL1 that regulates apoptosis (Figure 3, F-H). These results demonstrate that lncRNA DRAIR may regulate macrophage functions and promote antiinflammatory processes.

Next, we determined the effect of DRAIR silencing on the monocyte inflammatory phenotype. THP1 cells were transfected with siRNAs targeting DRAIR (siDR) or negative control siRNA (siNC) and, 48 hours later, treated with LPS $(100 \mathrm{ng} / \mathrm{mL})$ for 24 hours. qPCR analysis showed that siDR inhibited DRAIR expression and nearby $C P E B 2$, as well as $I L 1 R N$, but enhanced proinflammatory $I L 1 B$ expression (Figure 3, I-L). We also tested whether DRAIR gene silencing can downregulate ILIRN expression in THP1 macrophages. THP1 cells were transfected with $\mathrm{siDR}$ or siNC, followed by treatment with PMA $(20 \mathrm{ng} / \mathrm{mL})$, to induce macrophage differentiation. PMA treatment increased DRAIR expression, along with $I L I R N$ and CPEB2, but DRAIR gene silencing by siDR significantly inhibited both $C P E B 2$ and $I L 1 R N$ relative to siNC transfected cells (Figure 3 , $\mathrm{M}-\mathrm{O})$, further verifying that $D R A I R$ can positively regulate antiinflammatory genes in macrophages.

We also examined whether DRAIR gene silencing can enhance monocyte-endothelial cell (monocyte-EC) adhesion, a key indicator of inflammation. THP1 monocytes were transfected with siDR or siNC and, 48 hours later, treated with or without TNF- $\alpha(10 \mathrm{ng} / \mathrm{mL}$ for 3 hours $)$. Then, THP1 monocytes were fluorescently labeled with DAPI and incubated with primary human umbilical vein ECs plated in 24-well plates to perform adhesion assays. Monocyte-EC adhesion was significantly enhanced after DRAIR knockdown (Figure 3, P and Q; siNC versus siDR). However, TNF- $\alpha$-induced increases in monocyte-EC adhesion was not further enhanced by siDR (Figure 3, P and Q; siNC + TNF versus siDR + TNF). These results demonstrate that DRAIR gene silencing promotes the inflammatory phenotype in THP1 monocytes.

Phagocytosis is an important function of macrophages in normal and pathological conditions. Because $D R A I R$ is induced during macrophage differentiation and because phagocytosis-related pathways were enriched in differentially regulated genes inT2D monocytes (Supplemental Figure $1 G$ ), we tested if $D R A I R$ knockdown affects phagocytosis. We found that DRAIR knockdown with siDR significantly attenuated basal- and IL-4-induced phagocytosis of fluorescently labeled E. coli bioparticles in THP1 macrophages (Figure 3R). Furthermore, LPS also inhibited phagocytosis, and this was further inhibited after DRAIR knockdown (Figure 3R). Together, these data clearly demonstrate that DRAIR regulates antiinflammatory processes and key macrophage functions.

$D R A I R$ knockdown amplifies and DRAIR overexpression attenuates inflammatory genes in CD14+ monocytes. Next, we examined if DRAIR can elicit similar antiinflammatory effects in human CD14+ monocytes. We differentiated $\mathrm{CD} 14^{+}$monocytes isolated from nondiabetic human volunteers and transfected them with siDR or siNC. Two days after transfection, cells were treated with or without LPS, and gene expression was analyzed. siDR-mediated knockdown of DRAIR significantly enhanced LPS-induced expression of $I L 1 B, T N F$, and IL6 (Figure 4, A-D). Conversely, we examined the consequences of DRAIR overexpression by transfecting $\mathrm{CD} 14^{+}$monocytes with a plasmid vector pDRAIR expressing $D R A I R$ or control empty vector pcDNA3.1. DRAIR overexpression significantly attenuated LPS-induced $I L 1 B$, $T N F$, and IL6 expression (Figure 4, E-H), clearly demonstrating that DRAIR mediates antiinflammatory effects even in primary human monocytes.

DRAIR expression is induced by macrophage differentiation and by antiinflammatory cytokines via KLF4. Monocyte to macrophage differentiation plays an important role in inflammation and homeostasis. Therefore, we examined the expression of DRAIR in THP1 cells that were differentiated into macrophages by treatment with PMA (20 ng/mL). We found that expressions of DRAIR and CPEB2 were significantly increased in THP1 macrophages relative to monocytes at 24 hours after PMA treatment (Figure 5, A and B). In parallel, antiinflammatory $I L 1 R N$ was upregulated and proinflammatory $T N F$ was downregulated (Figure 5, C and D). Next, DRAIR expression was examined after treatment with IL-4 and IL-13, which are known to promote alternatively activated (M2) phenotype and antiinflammatory responses in macrophages. Both IL-4 and IL-13 (20 ng/mL, 24 hours) significantly induced DRAIR expression (Figure 5E). In parallel, CPEB2 and ILIRN were also upregulated by these 2 cytokines, whereas $T N F$ was downregulated (Figure 5, F-H). Treatment with a mixture of IL-4 and IL-13 had no further additive effects, suggesting that similar pathways regulate these genes (Figure 5, E-H). 


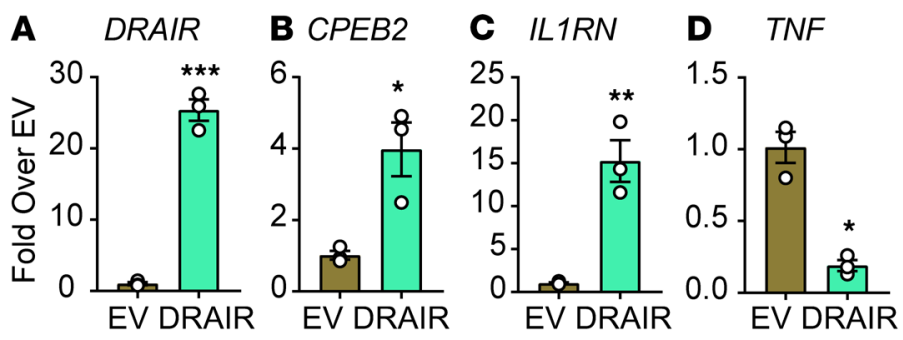

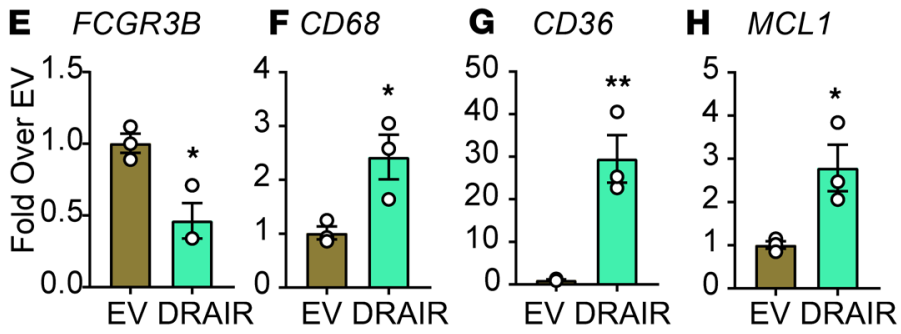
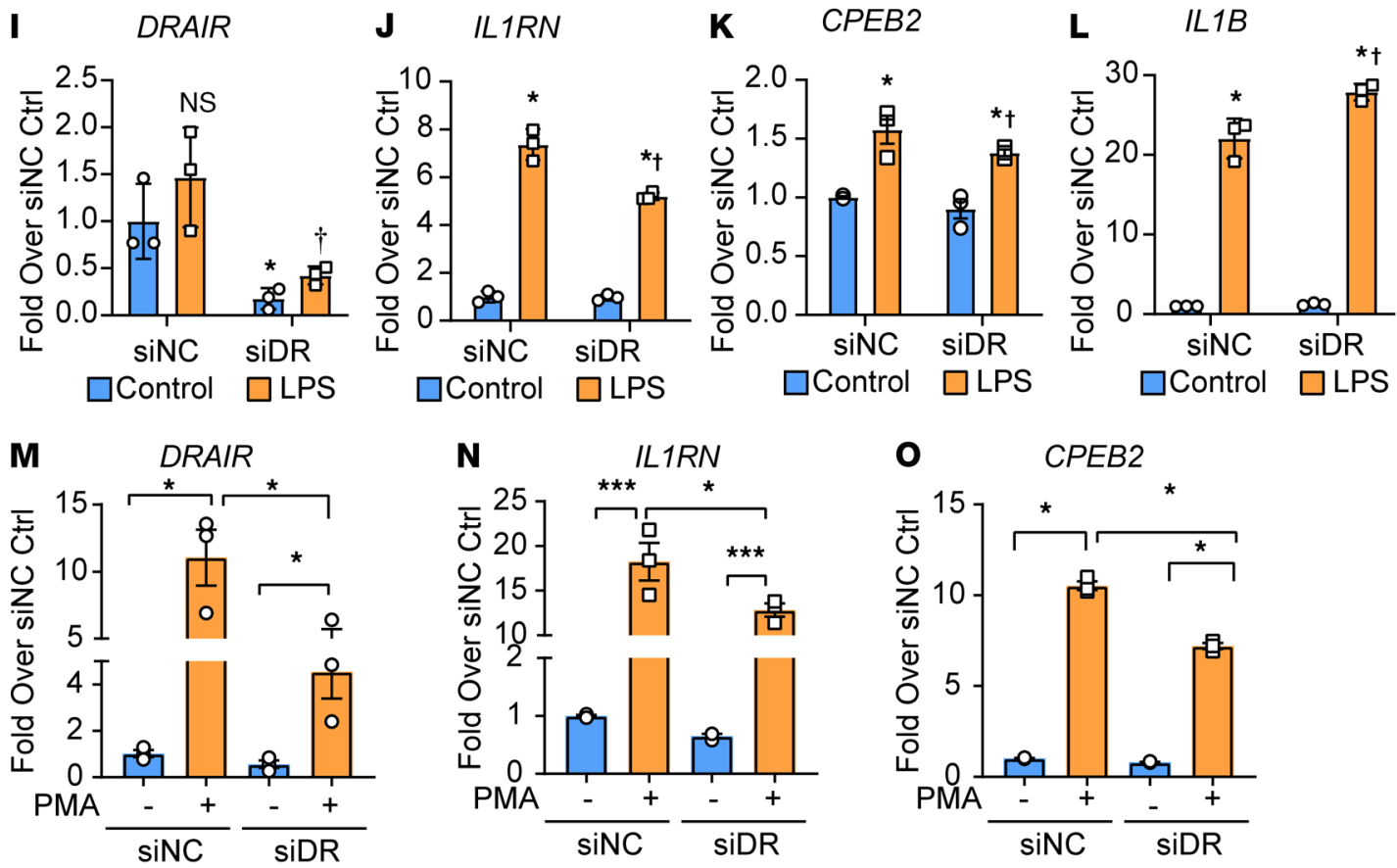

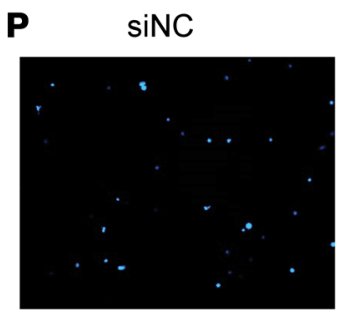

siDR

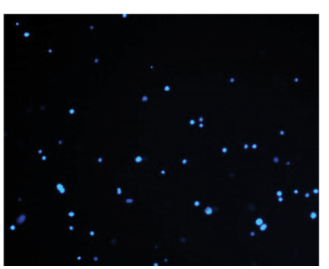

siNC+TNF
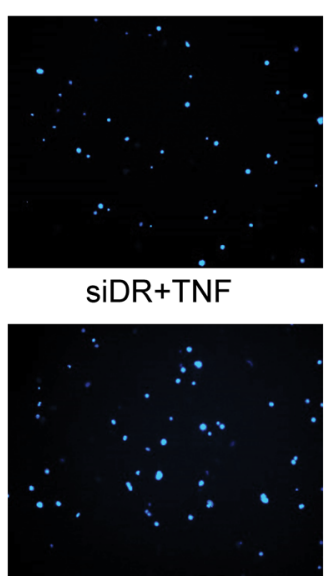

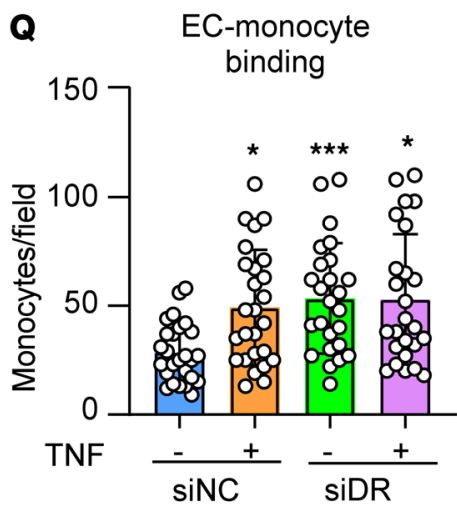

R Phagocytosis

ก 25

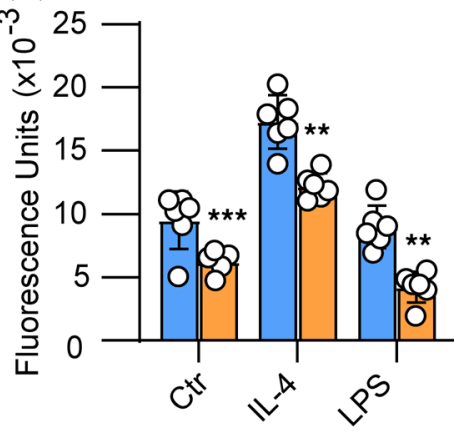

SiNC $\square$ siDR 
Figure 3. Effect of DRAIR overexpression or knockdown on proinflammatory gene expression and phenotype of THP1 monocytes. (A-H) Effects of DRAIR overexpression. qPCR analysis of indicated genes in THP1 cells transduced with lentivruses expressing DRAIR or a control vector (EV). ${ }^{*} P<0.05$; ${ }^{* *} P<0.01$; ${ }^{* *} P<0.001$, by $t$ test $(n=3)$. (I-L) Effects of DRAIR knockdown with siRNAs. THP1 cells were transfected with control siRNA (siNC) or siRNA targeting DRAIR (siDR). Two days later, cells were treated with or without with LPS $(100 \mathrm{ng} / \mathrm{mL})$ for 24 hours, and gene expression was analyzed by qPCR Results expressed as fold over siNC control. (M-0) qPCR analysis of THP1 cells transfected with siNC or siDR treated \pm PMA (20 ng/mL) for 24 hours. Results expressed as fold over siNC control (Ctrl). For I-0, ${ }^{*} P<0.05 ;{ }^{* * *} P<0.001$ versus siNC control and $+P<0.05$ versus siNC LPS as determined by 2 -way ANOVA $(\mathbf{I}-\mathbf{L})$, 1-way ANOVA (M-0), and Sidak's multiple-comparison test $(n=3)$. (P and $\mathbf{Q})$ THP1 cells transfected with siDR or siNC were treated \pm TNF- $\alpha(10$ ng/ $\mathrm{mL}, 3$ hours), labeled with DAPI, and used in monocyte-endothelial cell (monocyte-EC) adhesion assays. Images of bound monocytes were collected using a fluorescence microscope $(\mathbf{P})$. Total original magnification, $\times 100$. Bound monocytes from multiple wells/group were quantified using Image software (Q). ${ }^{*} P<0.05$; ${ }^{* *} P<0.001$ as determined by 1-way ANOVA and Sidak's multiple-comparison test (versus siNC control, $\left.n=25-29\right)$. (R) Phagocytosis assays were performed with fluorescently labeled $E$. coli bioparticles in THP1 macrophages transfected with siDR or siNC and treated with IL-4 (20 ng/mL) or LPS (100 $\mathrm{ng} / \mathrm{mL}$ ) for 24 hours. Results shown as fluorescence from phagocytosed particles. ${ }^{* *} P<0.01$; ${ }^{* *} P<0.001$ as determined by multiple unpaired $t$ tests, with correction for multiple comparisons using the Holm-Sidak method (versus siNC, $n=6$ ).

A TF motif search of the DRAIR promoter by TRANSFAC analysis revealed several binding sites for Kruppel-like family (KLF) members, including KLF4 (Supplemental Figure 5), a reported negative regulator of the macrophage inflammatory phenotype (31). Accordingly, transfection of THP1 cells with a KLF4 expression plasmid (pKLF4) significantly increased KLF4 expression and upregulated DRAIR and CPEB2 relative to empty vector (pCD) (Figure 5, I-K). Furthermore, ChIP assays with KLF4 antibody confirmed KLF4 binding at the DRAIR promoter but not at control PPIA promoter (Figure 5L). To further verify whether KLF4 regulates the DRAIR promoter, we constructed a reporter plasmid pDRluc in which LUC is expressed from the DRAIR promoter region $(-1064$ to $+39 \mathrm{bp})$ harboring the KLF4 binding site at -760 bp (Figure $5 \mathrm{M}$ ). Transient transfection with $\mathrm{pDR}$ luc showed that DRAIR promoter activity was inhibited by PA $(200 \mu \mathrm{M})$ but transactivated by IL-4 $(20 \mathrm{ng} / \mathrm{mL})$ and PMA that promotes differentiation of THP1 monocytes (THP) into macrophages (TMac) (Figure 5, N and O). Furthermore, cotransfection of pDRluc reporter plasmid with pKLF4 plasmid induced transactivation of the DRAIR promoter relative to $\mathrm{pCD}$, which was further enhanced after PMA treatment (Figure 5P). These results demonstrate that DRAIR expression is upregulated during macrophage differentiation and by antiinflammatory cytokines and that KLF4 plays a key role in its transcriptional activation.

CPEB2 gene silencing also promotes the inflammatory phenotype in THP1 monocytes. Because our data suggest that DRAIR could regulate the nearby gene CPEB2 that codes for CPEB2 protein, we examined if CPEB2 knockdown mimics the antiinflammatory effects of DRAIR. Transfection of THP1 cells with siRNA targeting CPEB2 (siCPEB2) inhibited expression of both CPEB2 and DRAIR, but upregulated IL1B and TNF compared with siNC transfected cells (Figure 6, A-D). Furthermore, CPEB2 knockdown in THP1 cells also enhanced both basal- and TNF- $\alpha$-induced monocyte-EC adhesion (Figure 6, E and F). These results suggest that CPEB2 may mediate some of the antiinflammatory effects of DRAIR in THP1 cells.

ChIRP analysis reveals DRAIR binding sites on chromatin. Interactions with chromatin and chromatin-interacting proteins are major mechanisms by which nuclear $\operatorname{lncRNAs}$ can regulate gene expression. Because $D R A I R$ is enriched in chromatin, we examined the interactions of DRAIR with chromatin by performing chromatin isolation by RNA purification (ChIRP) assays with THP1 nuclear lysates using biotinylated tiling oligonucleotide probes targeting the DRAIR RNA. Quantitative PCR (qPCR) confirmed specific recovery of DRAIR transcript but not control GAPDH in RNA recovered from ChIRP assays (Figure 7A). DNA recovered from ChIRP assays (ChIRP-DNA) was analyzed by DNA-seq (ChIRP-seq) to identify genome-wide interactions of DRAIR. ChIRP-seq analysis revealed 152 DRAIR binding sites (Dbs) on multiple chromosomes in THP1 cells (Supplemental Table 2). These Dbs were enriched at promoter, intronic, and enhancer regions on the chromatin (Figure 7B). Interestingly, 290 genes located nearby Dbs ( $\pm 250 \mathrm{~kb}$; Supplemental Table 3) were differentially expressed in our RNA-seq data from T2D monocytes (Figure 1B). IPA showed that these genes nearby Dbs are involved in inflammatory response, chemotaxis, and phagocytosis (Figure 7C). Transfac analysis of Dbs showed enrichment of key TFs involved in monocyte and macrophage functions, including members of the KLF family (Supplemental Figure 6).

To further understand the functions of DRAIR at the chromatin level, we examined the potential interaction of $\mathrm{Dbs}$ with other genomic regions using capture $\mathrm{Hi}-\mathrm{C}$ plotter $(\mathrm{CHiCP})$, a publicly available promoter capture Hi-C database in human cells (32). $\mathrm{CHiCP}$ analysis of human macrophages revealed interactions of Dbs with multiple genomic regions, suggesting their role in DRAIR mediated gene regulation. One of the Dbs was located in the intronic region of OPTC gene on chromosome 1, which we named OPTC-Dbs (Figure 7D). CHiCP analysis revealed that the OPTC-Dbs potentially interacts with a 

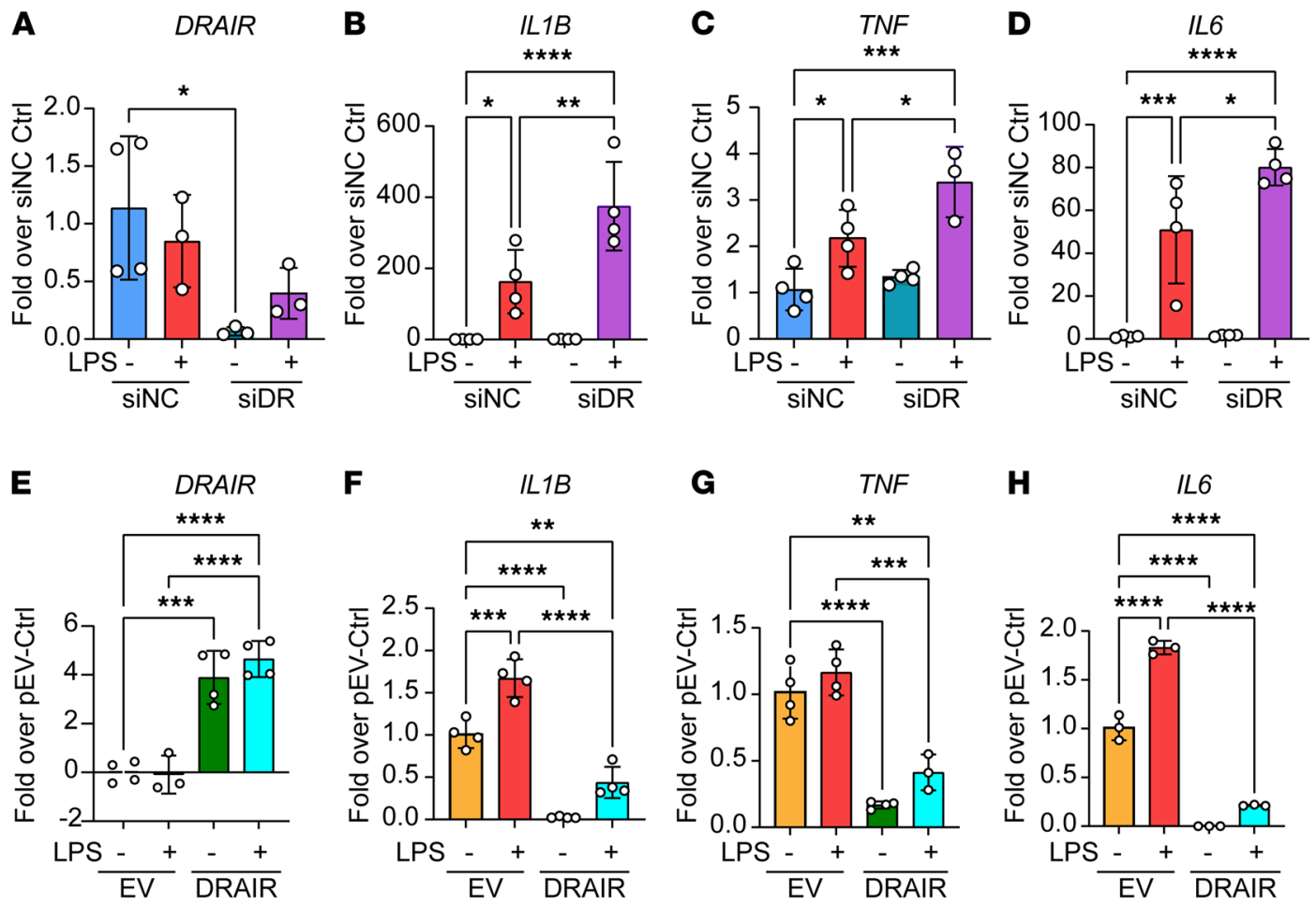

Figure 4. DRAIR regulates inflammatory phenotype of primary human $C D 14^{+}$monocytes. (A-D) DRAIR knockdown enhances inflammatory gene expression in human CD14+ monocytes. qPCR analysis of indicated genes in CD14+ monocytes transfected with siDR or siNC. Two days after transfection, cells were treated with or without LPS $(100 \mathrm{ng} / \mathrm{mL})$ for 24 hours and gene expression analyzed by qPCR. Results expressed as fold over siNC control (Ctrl). ${ }^{*} P<0.05$; ${ }^{* *} P<0.01$; ${ }^{* *} P<0.001 ;{ }^{* * *} P<0.0001$ versus siNC-LPS, as determined by 1 -way ANOVA followed by Sidak's multiple-comparison tests ( $n=$ 4). Similar results were obtained with $C D 14^{+}$monocytes isolated from 2 other volunteers. (E-H) DRAIR overexpression inhibits inflammatory phenotype in primary human $\mathrm{CD} 14^{+}$monocytes. CD14+ monocytes transfected with plasmid pDRAIR expressing DRAIR (DRAIR) or empty vector pcDNA3.1 (EV) were treated with LPS $(100 \mathrm{ng} / \mathrm{mL})$ for 24 hours, and expression of indicated genes was analyzed by qPCR $(n=4) .{ }^{* *} P<0.01 ;{ }^{* *} P<0.001$; ${ }^{* * * *} P<0.0001$ as determined by 1-way ANOVA and Sidak's multiple-comparison tests $(n=4)$.

genomic region $362 \mathrm{~kb}$ away on the same chromosome with a Capture Hi-C Analysis of Genomic Organization (CHiCAGO) score of $>5$. This Dbs interacting region is upstream of CHIT1 and CHI3L genes (Figure 7E), which regulate the macrophage M2 phenotype (33).

Using candidate ChIRP-qPCR, we validated the interaction between DRAIR and the OPTC-Dbs in THP1 monocytes. Moreover, this interaction was abolished by RNase treatment of THP1 lysates, confirming the specificity of the interaction (Figure 7F). We next examined whether DRAIR regulates CHITI and $C H I 3 L$ genes. qPCR analysis showed that DRAIR overexpression significantly upregulated CHITI and $C H I 3 L$, but not $\operatorname{lnc} 01136$, a lncRNA expressed in the nearby region (Figure 7, G-I), suggesting that $D R A I R$ may regulate these genes through interaction at OPTC-Dbs in monocytes. In addition, candidate ChIRP-qPCR also demonstrated DRAIR interaction with the promoter of adjacent CPEB2 (Figure 7F), suggesting a role for chromatin interaction in the regulation of nearby CPEB2 gene by DRAIR. These results indicate that $D R A I R$ interactions with chromatin may play important roles in the regulation of proximally and distally located monocyte/macrophage genes.

DRAIR interacts with G9a histone methyltransferase and controls repressive histone modifications at target gene promoters. We next used ChIRP followed by mass spectrometry (ChIRP-MS) to explore whether DRAIR also regulates gene expression via interactions with chromatin modifying enzymes/proteins. Because endogenous DRAIR is not abundant in THP1 monocytes cells, we used a THP1 cell line stably overexpressing $D R A I R$ for these experiments. ChIRP was performed using biotinylated tiling oligonucleotide probes complementary to DRAIR, and $L U C$ transcript (negative control). qPCR of RNA recovered from ChIRP samples (ChIRP-RNA) showed enrichment of DRAIR RNA only with DRAIR probes but not with LUC probes, confirming specificity (Figure 8A). Following ChIRP, nucleic acid-protein complexes were fractionated by SDS-PAGE $(4 \%-15 \%)$, and the proteins captured using DRAIR or Luc probes were analyzed by MS. ChIRP-MS analysis revealed that DRAIR interacts with several nuclear proteins including histone 

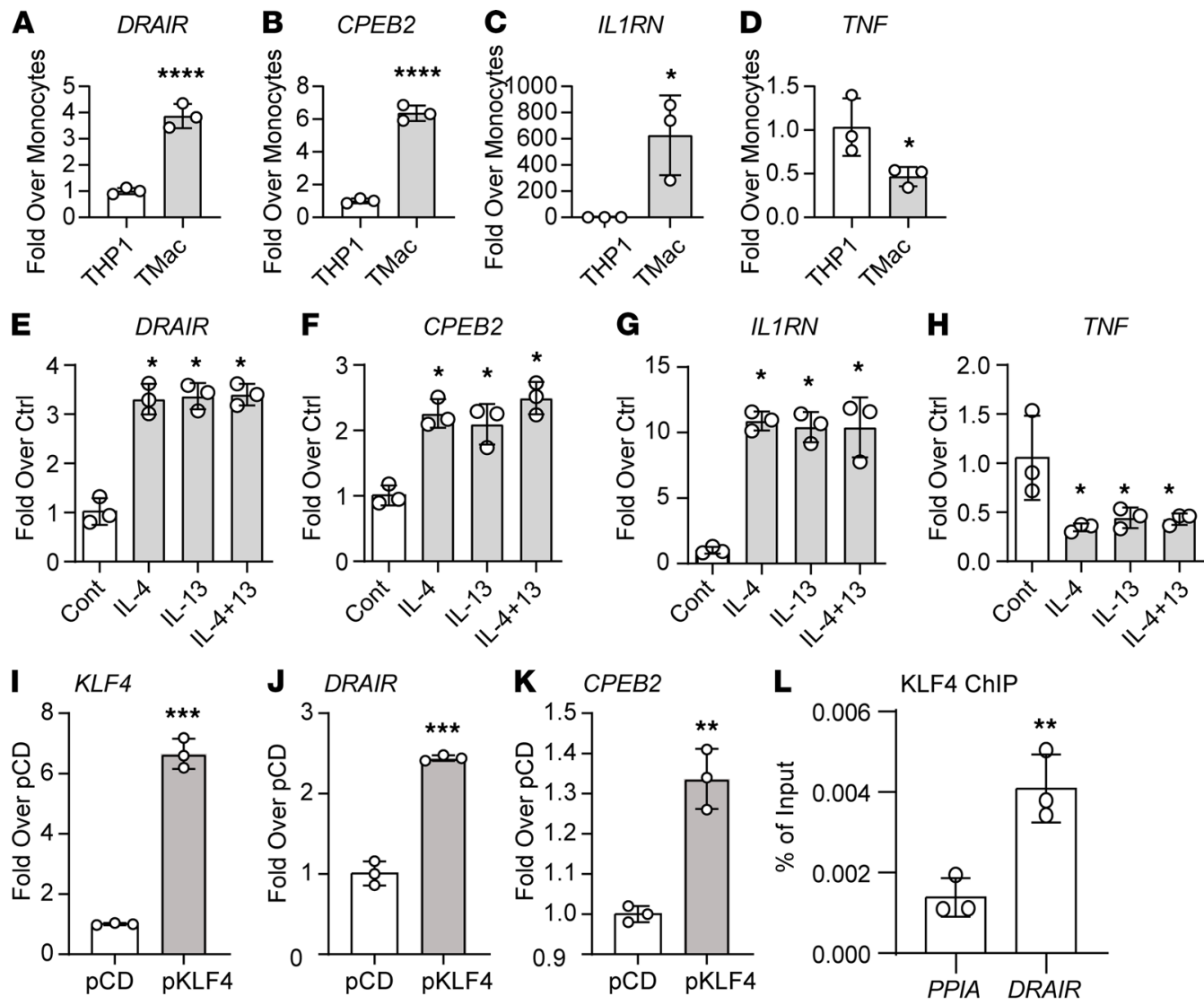
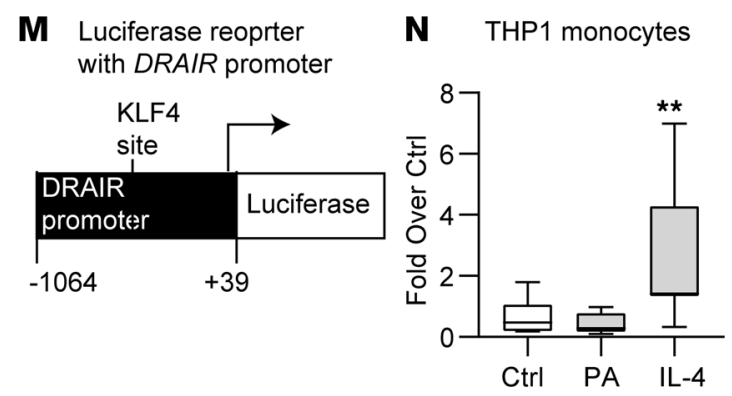
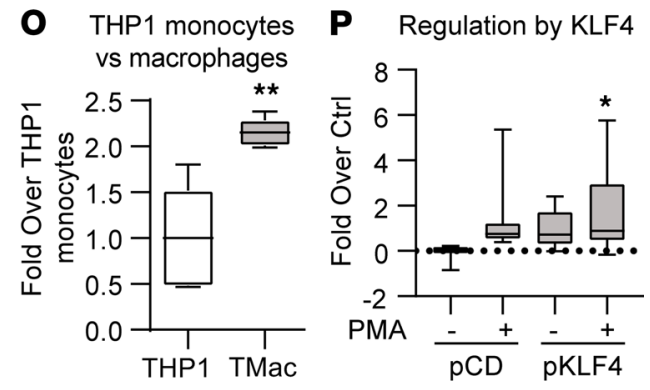

Figure 5. DRAIR is regulated during macrophage differentiation and by antiinflammatory cytokines. (A-D) qPCR analysis of indicated genes in THP1 monocytes (THP1) before and after differentiation into macrophages (TMac) with PMA $(20 \mathrm{ng} / \mathrm{mL})$ for 24 hours. ${ }^{*} P<0.05 ;{ }^{* * *} P<0.0001$ as determined by unpaired $t$ test $(n=3)$. (E-H) Expression of indicated genes in THP1 macrophages treated with IL-4 or IL-13 or a combination of both $\left(20 \mathrm{ng} / \mathrm{mL}\right.$ each). ${ }^{*} P<0.05$ $(n=3)$ as determined by 1-way ANOVA and Dunnett's multiple comparisons tests. (I-K) Gene expression analysis in THP1 cells transiently transfected with control pcDNA3.1 (pCD) or KLF4 expression (pKLF4) plasmids. Gene expression analyses were performed 48 hours after transfection. ${ }^{* *} P<0.01$; ${ }^{* *} P<0.001$ versus $p C D$ as determined by unpaired $t$ test $(n=3)$. (L) ChIP-qPCR analysis of ChIP assays with KLF4 antibody with indicated promoter primers $\left({ }^{* *} P<0.01\right.$ versus PPIA promoter, $n=3$ ). (M) Schematic of the reporter plasmid (pDRluc) with DRAIR promoter cloned upstream of firefly luciferase reporter gene. KLF4 site (-760) in DRAIR promoter (not to scale). (N-P) Luciferase activities with THP1 cells cotransfected with pDRluc and internal control Renilla luciferase. In addition, plasmids pKLF4 and pCDNA3.1 were also cotransfected in $\mathbf{P}$. One day after transfection, cells were treated as indicated for $\mathbf{2 4}$ hours. Luciferase activities are reported as fold over controls. In N, Ctrl, control; PA, palmitic acid (200 $\mu \mathrm{M})$; IL-4, $20 \mathrm{ng} / \mathrm{mL}$; in 0, PMA-PMA $20 \mathrm{ng} / \mathrm{mL}$. ${ }^{*} P<0.05$, ${ }^{*} P<0.01$ as determined by 1-way ANOVA followed by Dunnett's multiple-comparison test ( $\mathbf{N}$ and $\mathbf{P}, n=$ 10-13) and unpaired $t$ test for $\mathbf{0}(n=5)$.

modifying enzymes, transcription repressors, and enhancer interacting proteins (Supplemental Figure 7A). STRING analysis (34) of DRAIR-interacting proteins showed that networks of these proteins were associated with GO biological processes and molecular functions related to histone methylation, transcription, chromatin organization, and gene expression (Figure 8B and Supplemental Figure 7, B and C). 

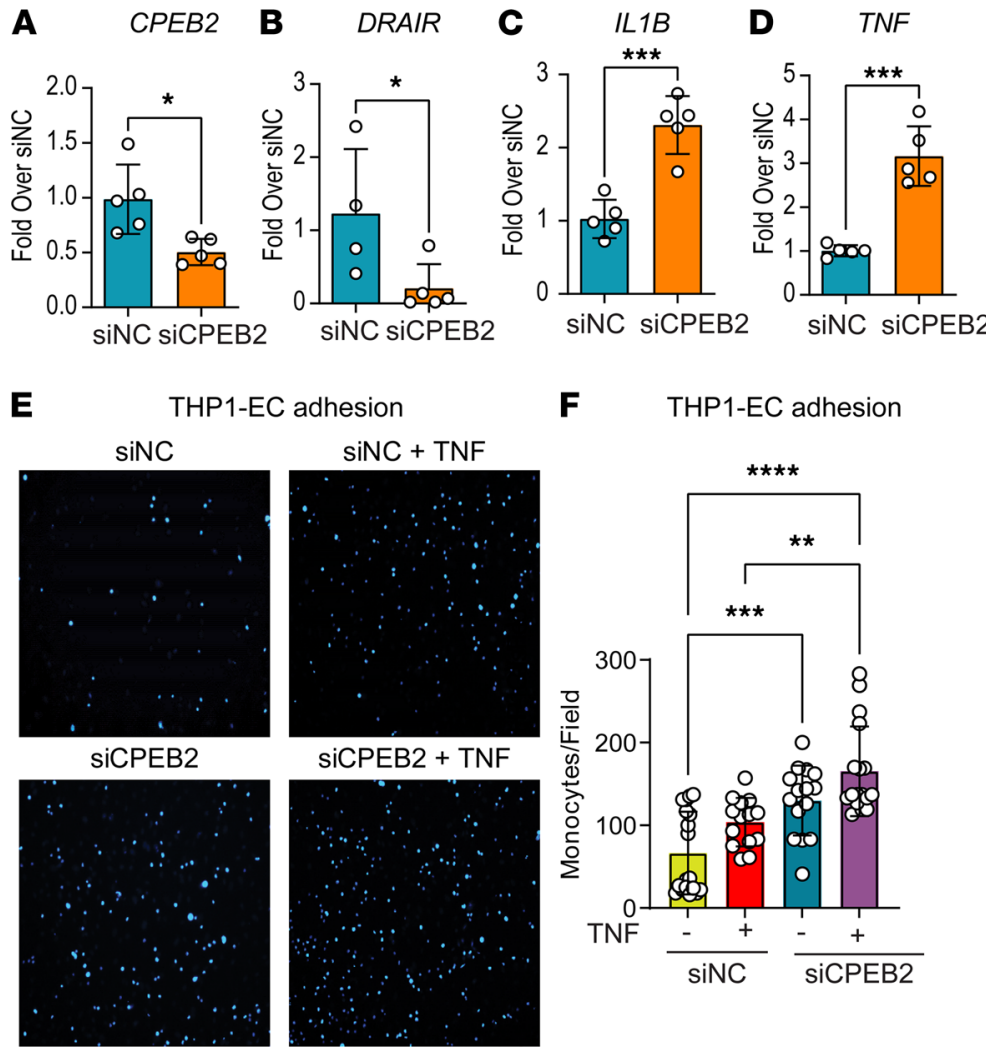

$\mathbf{F}$

THP1-EC adhesion siNC + TNF
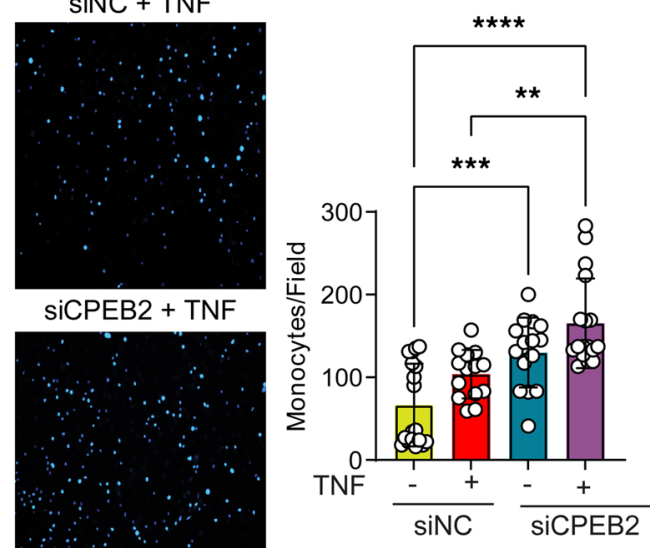

Figure 6. CPEB2 knockdown also promotes inflammatory phenotype in THP1 monocytes. (A-D) Expression of indicated genes was analyzed by qPCR in THP1 cells transfected with siNC and siRNA targeting CPEB2 (siCPEB2). ${ }^{*} P<$ 0.05 ; ${ }^{* *} P<0.001(n=5)$ versus untreated siNC as determined by unpaired $t$ test. (E and $\left.\mathbf{F}\right)$ Images and quantification of monocyte-endothelial cell (monocyte-EC) adhesion assays. THP1 monocytes transfected with siCPEB2 or siNC were treated \pm TNF- $\alpha(10 \mathrm{ng} / \mathrm{mL}, 3$ hours), fluorescently labeled with DAPI, and incubated with EC plated in 24-well plates. EC monolayers were washed with PBS, and images were collected using a fluorescence microscope. Total original magnification, $\times 100$. Bound monocytes (blue color spots) were counted using Imagel software. ${ }^{* *} P<0.01$, ${ }^{* * *} P<$ $0.001,{ }^{* * *} P<0.0001$ versus untreated siNC and versus untreated siCPEB2 as determined by 1 -way ANOVA and Sidak's multiple-comparison test $(n=16-19)$.

These results suggest that $D R A I R$ interaction with chromatin modifying protein networks may regulate epigenetic mechanisms involved in $D R A I R$-mediated gene regulation.

To examine this further, we validated $D R A I R$ interaction with 2 proteins identified by ChIRP-MS, namely histone methyl transferases G9a (EHMT2) and SUV39H1 (KMT1A), which mediate the repressive histone modifications histone $\mathrm{H} 3$ lysine-9 dimethylation (H3K9me2) and $\mathrm{H} 3 \mathrm{~K} 9 \mathrm{me}$, respectively. Previous studies showed dysregulation of $\mathrm{H} 3 \mathrm{~K} 9 \mathrm{me} 2$ and $\mathrm{H} 3 \mathrm{~K} 9 \mathrm{me} 3$ by hyperglycemia and diabetes in monocytes and vascular cells $(11,35)$. DRAIR interaction with G9a was validated using RNA pulldown and RNA IP (RIP) assays. RNA pulldown was performed on nuclear extracts from THP1 cells using a biotinylated $D R A I R$ sense probe and DRAIR antisense (DRAIR-AS) probe as negative control. Western blot analysis of RNA pulldown proteins revealed that G9a strongly interacts with DRAIR probe but not with DRAIR-AS probe (Figure 8C). However, SUV39H1 protein was not detected after RNA pulldown (data not shown). Furthermore, RIP also confirmed enrichment of DRAIR with G9a antibody but not with IgG or SUV39H1 antibody (Figure 8D).

Next, we determined the effect of $D R A I R$ overexpression on G9a occupancy and enrichment levels of the corresponding repressive histone modification $\mathrm{H} 3 \mathrm{~K} 9 \mathrm{me} 2$ at candidate $D R A I R$ target genes shown in Figure 3, B-H. ChIP-qPCRs showed that H3K9me2 levels were significantly reduced at IL1RN, CPEB2, and $M C L 1$ promoters (Figure 8, E-G) in DRAIR-overexpressing cells versus empty vector pcDNA3.1 transfected cells. These candidate genes were upregulated in DRAIR-overexpressing cells (Figure 3). However, H3K9me2 levels were not altered at the FCGR3B promoter (Figure 8H). Furthermore, ChIP assays with G9a antibody showed that, in parallel, G9a occupancy was also significantly reduced at Il1RN, CPEB2, and $M C L 1$ promoters but not at the FCGR3B promoter (Figure 8, I-L). Because H3K9me2 and G9a were not 
A ChIRP RNA

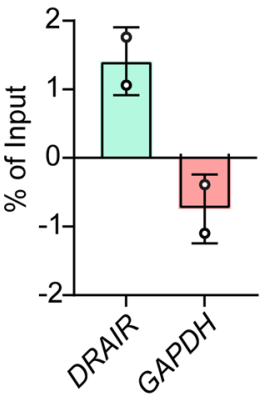

B Peak Distribution

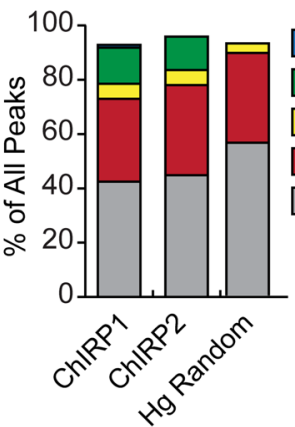

C

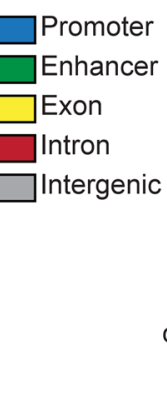

IPA of ChIRP Genes

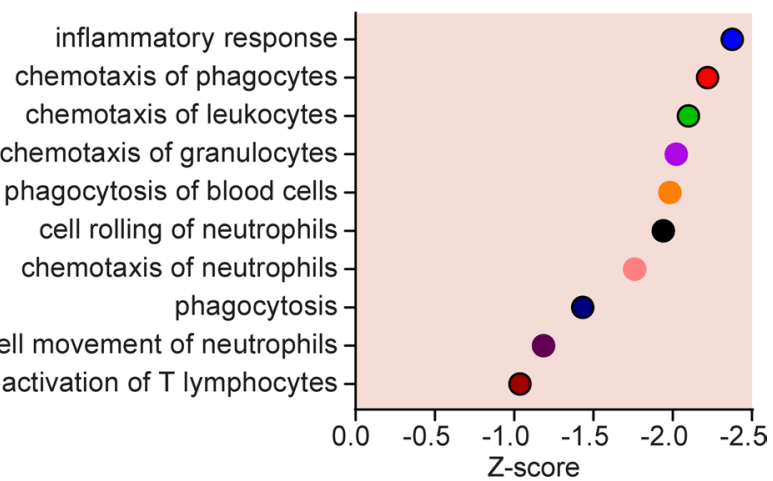

D

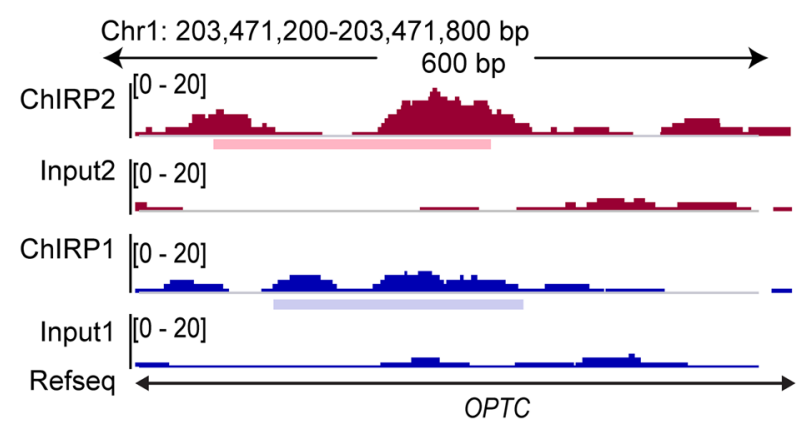

$\mathbf{F}$

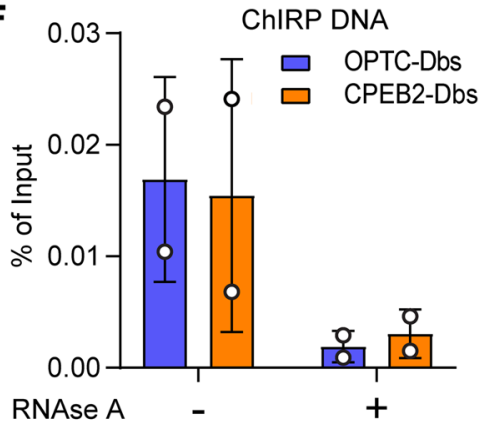

G

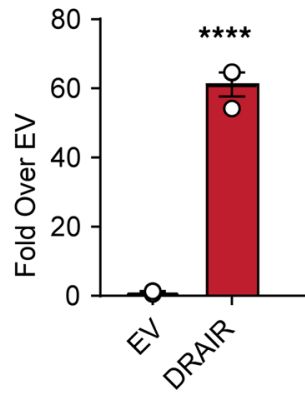

E Interaction of OPTC-Dbs with CHIT1

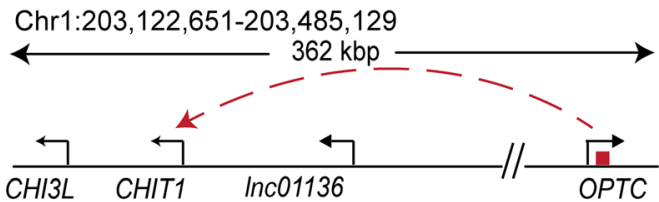

Figure 7. ChIRP analysis reveals DRAIR binding sites on chromatin. (A) qPCR analysis of RNA from ChIRP assays with indicated primers. Percentage of input values from 2 experiments were log transformed. ChIRP assays were performed using biotinylated DRAIR antisense oligonucleotides in THP1 cells. RNA-recovered from ChIRP assays was analyzed by qPCR and DNA by DNA sequencing (ChIRP-seq). (B) Genomic distribution of DRAIR binding sites (Dbs) identified from ChIRP-seq analysis. (C) GO terms enriched in genes nearby ( $\pm 250 \mathrm{~kb}$ ) Dbs as determined by IPA. (D and E). Schematic of Dbs in the intronic region of OPTC gene (OPTC-Dbs) (D) and its potential interaction with upstream region of CHIT1 gene (E) identified using Hi-C plotter (CHiCP) tool. ChIRP1 and ChIRP2 in $\mathbf{B}$ and $\mathbf{D}$ refer to duplicates. (F) ChIRP-qPCR analysis of ChIRP-DNA using primers for indicated DRAIR binding sites. THP1 cell lysates were treated \pm pancreatic ribonuclease $A$ (RNase A), and ChIRP assays were performed with biotinylated DRAIR probes. RNase A-treated samples served as negative controls. Percentage of input values from 2 experiments were log transformed. (G-I) qPCR analysis of indicated genes in THP1 cells overexpressing DRAIR versus control vector (EV). ${ }^{*} P<0.05 ;{ }^{* * *} P<0.0001$ versus EV as determined by unpaired $t$ test $(n=3)$.

altered at the FCGR3B promoter and because ChIRP-MS showed interaction of DRAIR with EED protein, which is a part of the polycomb repressive complex-2 (PRC2) that regulates H3K27me3 repressive mark (36), we tested the impact of DRAIR overexpression on H3K27me3. Indeed, ChIP assays showed that $D R A I R$ overexpression significantly increased $\mathrm{H} 3 \mathrm{~K} 27 \mathrm{me} 3$ at the FCGR $3 B$ promoter (Figure $8 \mathrm{M}$ ), whose expression was decreased under these conditions (Figure $3 \mathrm{E}$ ). These results suggest that DRAIR upregulates key target genes in part via sequestration of G9a, and downregulates other genes in part via activation of the PRC2 repressive complex in monocytes, thus implicating epigenetic mechanisms of action.

Interestingly, our RNA-seq data reveal that G9a gene (EHMT2) expression is upregulated in T2D monocytes versus controls ( $\log _{2}$-fold $=1.15, \mathrm{FDR}=0.047, n=5$ each). Therefore, we examined if G9a protein 

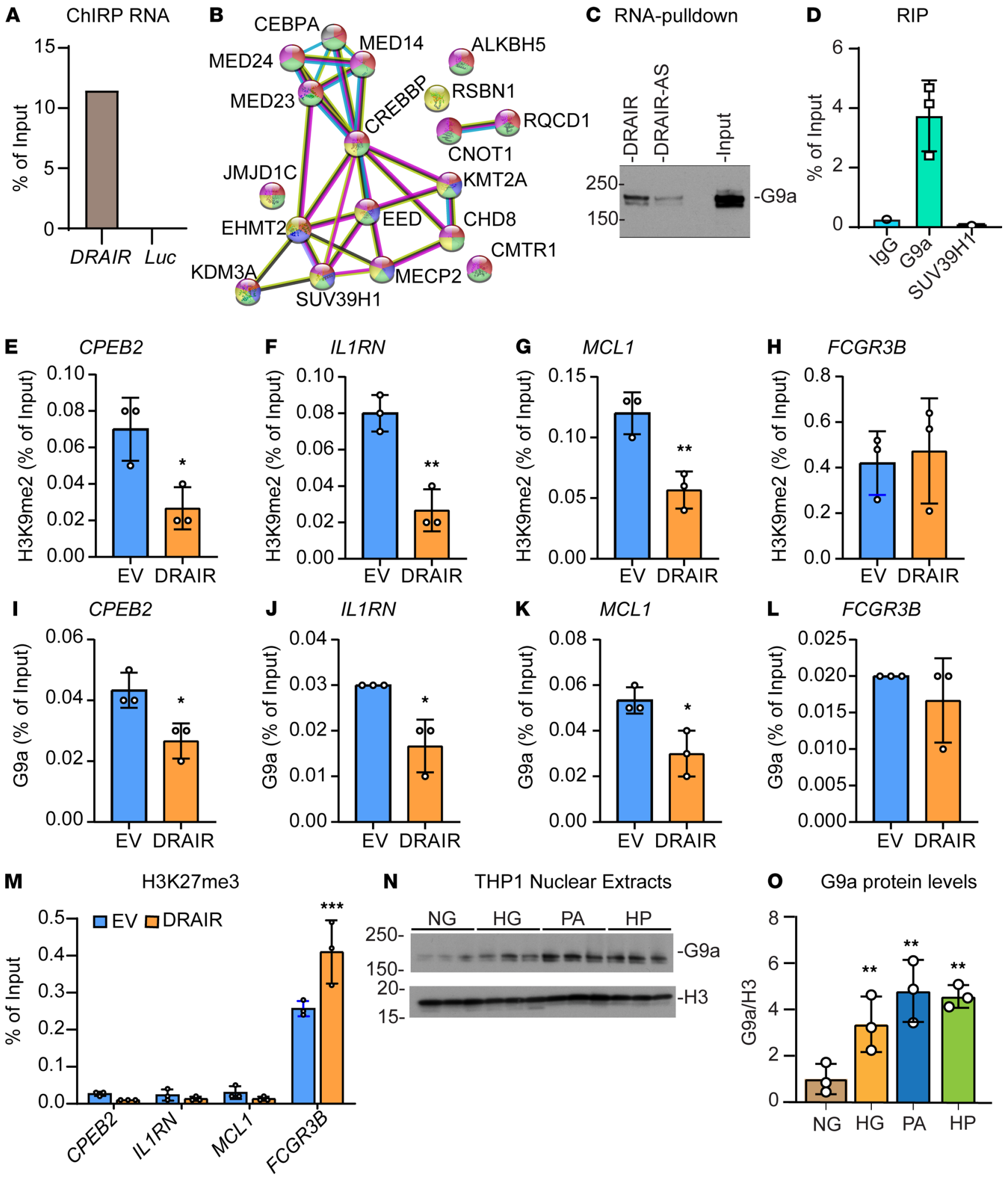

- G9a protein levels

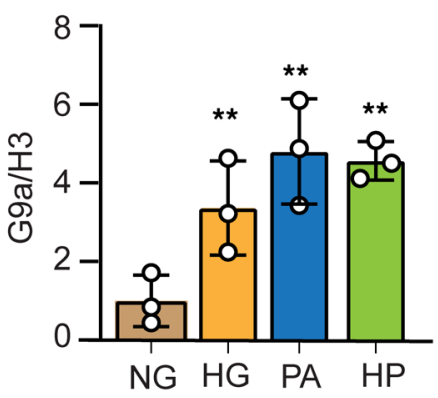

Figure 8. DRAIR interacts with G9a and alters levels of repressive histone modifications. (A) qPCR of RNA recovered from ChIRP with DRAIR probes or negative control luciferase (LUC) probes. Cell lysates from THP1 monocytes overexpressing DRAIR were subjected to ChIRP assays with biotinylated DRAIR probes and negative control biotinylated LUC probes. ChIRP complexes were captured on streptavidin beads, and RNA from an aliquot of beads was analyzed by qPCR with DRAIR primers. (B) The nucleic acid-protein complexes from ChIRP were fractionated on SDS-PACE and subjected to mass spectrometry to identify proteins interacting with DRAIR probes. STRING analysis of indicated DRAIR-interacting proteins identified by ChIRP mass spectrometry (Supplemental Figure 7). Colors represent GO biological functions shown in Supplemental Figure 7. (C) Immunoblotting of proteins from RNA pulldown assays using DRAIR and DRAIR antisense (DRAIR-AS) probes with G9a antibody. (D) qPCR analysis of RNA recovered after RNA IP with indicated antibodies. (E-M) ChIP-qPCR analysis of DNA recovered from ChIP assays using lysates from THP1 cells overexpressing DRAIR and empty vector pcDNA3.1 (EV) with antibodies against H3K9me2 (E-H), G9a (I-L), and H3K27me3 (M) using indicated gene 
promoter primers. ( $\mathbf{N}$ and $\mathbf{0}$ ) Immunoblotting of THP1 nuclear extracts with indicated antibodies ( $\mathbf{N}$ ) and quantification of 69 a in nuclear extracts (0). THP1 cells were treated with NG (5.5 mM glucose) and HC (25 mM glucose) for 72 hours. Palmitic acid (PA, $200 \mu M)$ was also added in the final 24 hours to NG- (PA) and HG-treated (HP) cells. ${ }^{*} P<0.05$; ${ }^{* *} P<0.01 ;{ }^{* *} P<0.001(n=3)$ as determined by unpaired $t$ test $(\mathbf{E}-\mathrm{L})$ and 2 -way ANOVA with Sidak's (M), and 1-way ANOVA with Dunnett's (0) multiple-comparison testing.

levels were altered in THP1 monocytes under diabetic conditions. Immunoblot analysis of nuclear extracts from THP1 monocytes treated with HG or PA alone or with HP showed significant increase in G9a protein levels (Figure 8, N and O). Furthermore, EHMT2 gene silencing with siRNA (siG9a) significantly increased expression of DRAIR target genes IL1RN, CPEB2, and MCL1 relative to nontargeting siRNA (siNTC) in THP1 cells (Supplemental Figure 8). These data suggest that downregulation of DRAIR and upregulation of G9a in T2D might work together, at least in part, to repress key antiinflammatory genes.

Mouse orthologous Drair is downregulated in macrophages from T2D mice and regulates the inflammatory phenotype. To evaluate putative conservation across species, we next examined expression and function of mouse orthologous Drair. Using liftOver, we found that an annotated lncRNA Gm7854 on mouse chromosome 5 is expressed as a divergent transcript near the mouse Cpeb2 gene (Figure 9A) similar to the human transcript. We designated Gm7854 as mouse Drair and examined its expression in peritoneal macrophages (PMs) from $d b / d b$ mice, a well-known mouse model of T2D. qPCR showed that Drair and nearby Cpeb2 were significantly downregulated in PMs from $d b / d b$ mice versus nondiabetic control $d b /+$ mice (Figure 9, B and C), similar to human DRAIR. We next examined the outcome of targeting mouse Drair with specific locked nucleic acid-modified (LNA-modified) GapmeRs. Transfection of the mouse RAW264.7 macrophage cell line with 3 GapmeRs (DRGa, DRGb, and DRGc) targeting different regions of Drair showed that only DRGb significantly inhibited Drair expression relative to control GapmeR NCG (Figure 9D). Moreover, Drair knockdown inhibited Cpeb2 but upregulated Tnf and Illb in RAW264.7 macrophages (Figure 9, E-G) compared with NCG. Interestingly, the expression of Illrn was also increased (Figure 9H), possibly as a feedback response to increased inflammation.

Next, we examined the effect of Drair knockdown in vivo on the macrophage inflammatory phenotype. Because, Drair is already downregulated in diabetic $d b / d b$ mouse macrophages, we examined whether Drair knockdown in nondiabetic C57BL/6 mice can elicit a diabetic-like inflammatory phenotype in macrophages. C57BL/6 mice were injected i.p. with thioglycollate (3\%) to induce inflammation and subsequently injected i.p. with 2 doses $(5 \mathrm{mg} / \mathrm{kg}$ ) of in vivo grade Drair GapmeR DRGb or negative control GapmeR NCG (Figure 9I). Gene expression analyzed in PMs 24 hours after the second dose showed that GapmeR DRGb significantly reduced Drair expression relative to GapmeR NCG-injected mice (Figure 9J). Furthermore, Drair knockdown inhibited the expression of nearby Cpeb2 (Figure 9K) and, in parallel, increased the expression of proinflammatory genes $T n f$, Illb, and Il6 (Figure 9, L-N), whereas Il1rn expression was not altered (Figure 9O). Altogether, these data demonstrate that Drair has similar antiinflammatory functions in mice and human macrophages

\section{Discussion}

Here, we show that several antiinflammatory genes and antiproliferative genes are downregulated in monocytes from T2D individuals relative to healthy controls and that this downregulation may contribute to increased inflammatory phenotype and monocyte numbers. Notably, we demonstrate that levels of a key IncRNA, DRAIR, were significantly lower in monocytes from T2D subjects, as well as in primary human monocytes from nondiabetic volunteers treated with HP. Our data show that DRAIR can promote antiinflammatory phenotype in monocytes/macrophages and that DRAIR expression is regulated by the TF KLF4, which was previously identified as a negative regulator of macrophage inflammation (31). Notably, we found that DRAIR increases key target antiinflammatory genes, such as ILIRN and CPEB2, in monocytes through potentially novel epigenetic derepression mechanisms. In addition, we found that the mouse ortholog Drair is downregulated in macrophages of T2D mice and that its knock down in vivo in nondiabetic mice increases expression of inflammatory genes in macrophages. These data suggest that downregulation of lncRNAs, such as DRAIR, that control endogenous antiinflammatory networks may contribute to key mechanisms leading to chronic inflammatory phenotype of monocytes in T2D and its complications.

Interestingly, IPA of downregulated genes in T2D (identified from RNA-seq) showed enrichment of NF- $\mathrm{BB}$ and ROS pathways. Because many of the downregulated genes, including ILIRN, NFKBIA, TNFAIP3 (A20), and NFE2L2 (NRF2) are well-known mediators of antiinflammatory and antioxidative 
A
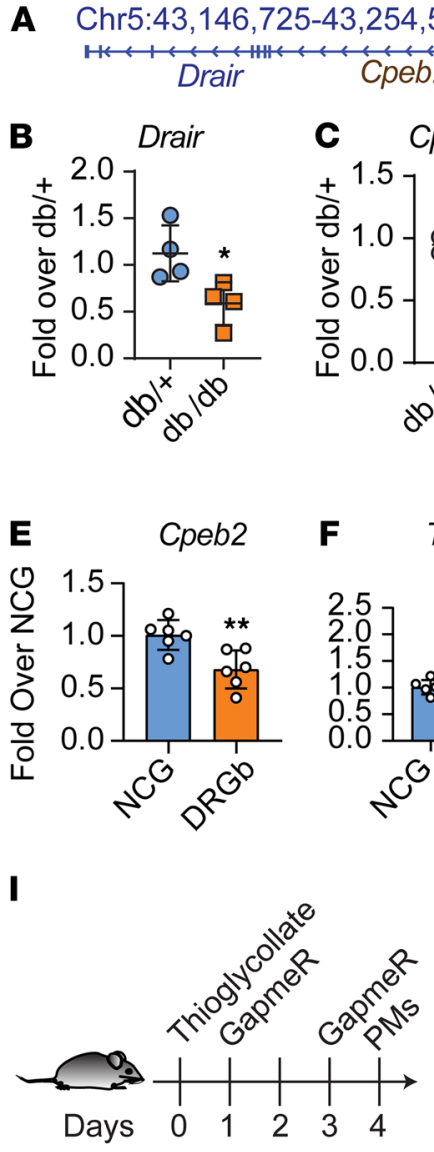

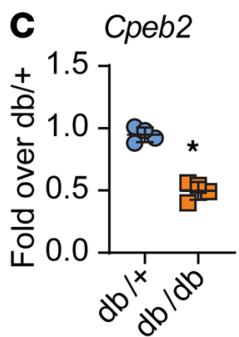

D Drair Gapmers

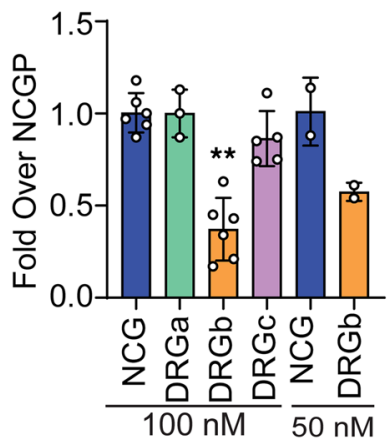

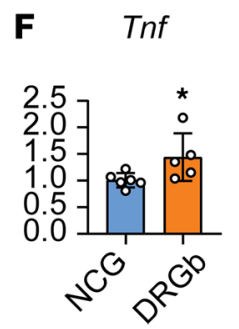
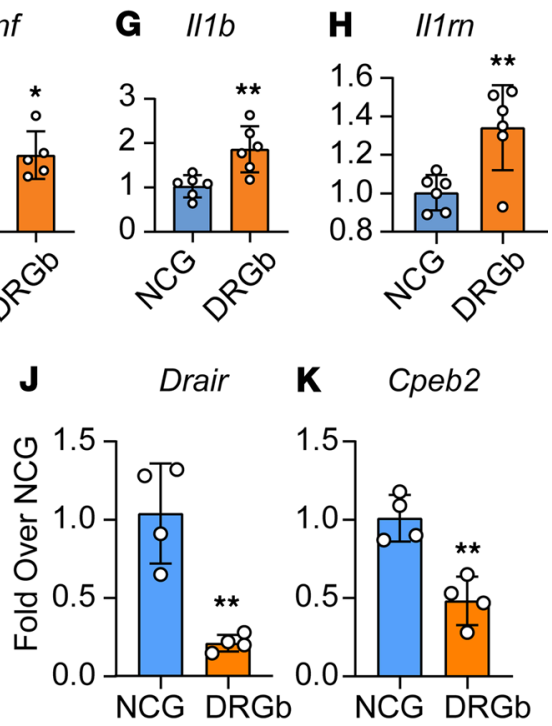

K $\quad$ Cpeb2
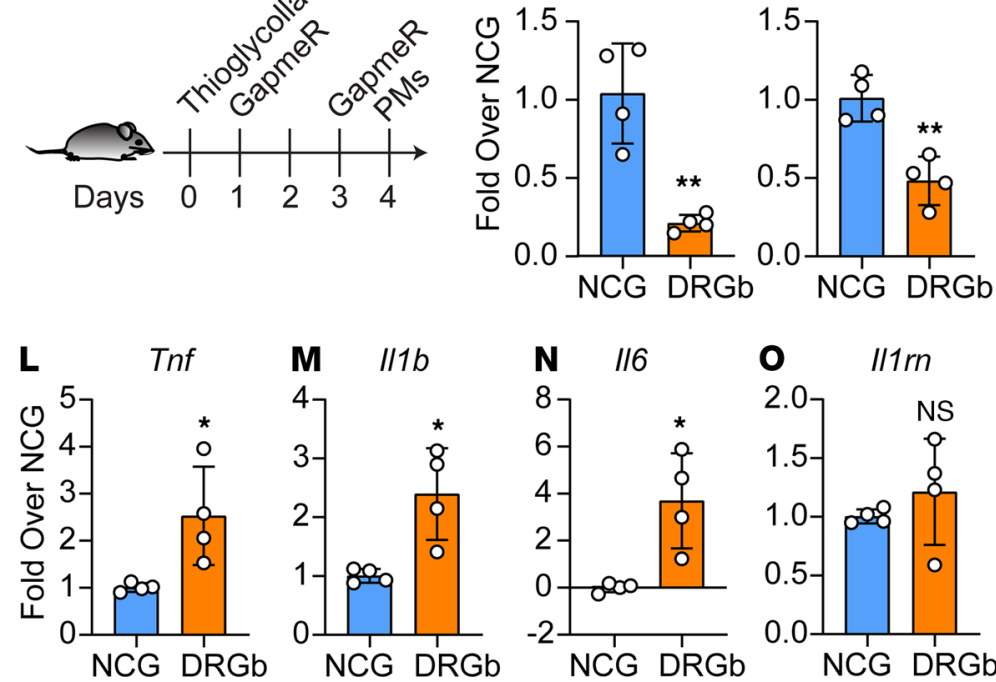

Figure 9. Mouse orthologous Drair is downregulated in macrophages from T2D mice and regulates inflammatory phenotype in macrophages. (A) Genomic organization of Drair and Cpeb2 in the mouse genome ( $\mathrm{mm} 9)$. (B and $\mathbf{C})$ qPCR analysis of Drair in peritoneal macrophages (PMs) from type2 diabetic $d b / d b$ mice versus genetic control $d b /+$ mice. ${ }^{*} P<0.05$ as determined by unpaired $t$ test $(n=4)$. (D) qPCR analysis of RAW cells transfected with control (NCG) and indicated Drair GapmeRs (DRGa, DRGb, and DRGc) $(n=2-6)$. (E-H) qPCR analysis of indicated genes after Drair knockdown with DRGb GapmeR (DRGb) versus NCG GapmeR in RAW cells ${ }^{*} P<0.05$; ${ }^{*} P<0.01$ (paired $t$ test). (I) Experimental design for Drair knockdown in C57BL/6 mice with GapmeRs (NCG or DRGb). (J-0) Gene expression analysis in PMs from C57BL/6 mice treated with NCG or Drair (DRGb) GapmeRs. ${ }^{*} P<0.05$; ${ }^{* *} P<0.01$ versus NCG as determined by unpaired $t$ test $(n=4)$.

stress mechanisms (Figure 1C), their downregulation can augment inflammatory phenotypes. In addition, downregulation of tumor suppressors such as PTEN can lead to monocytosis, thus further increasing the number of inflammatory monocytes. Evidence shows that monocytosis and enhanced infiltration of inflammatory monocytes in diabetes is linked with increased atherosclerosis burden (6). Together, these data show that downregulation of key antiinflammatory and tumor suppressor/antiproliferative genes in T2D might lead to increased inflammatory and proliferative state of monocytes implicated in diabetes vascular complications. 

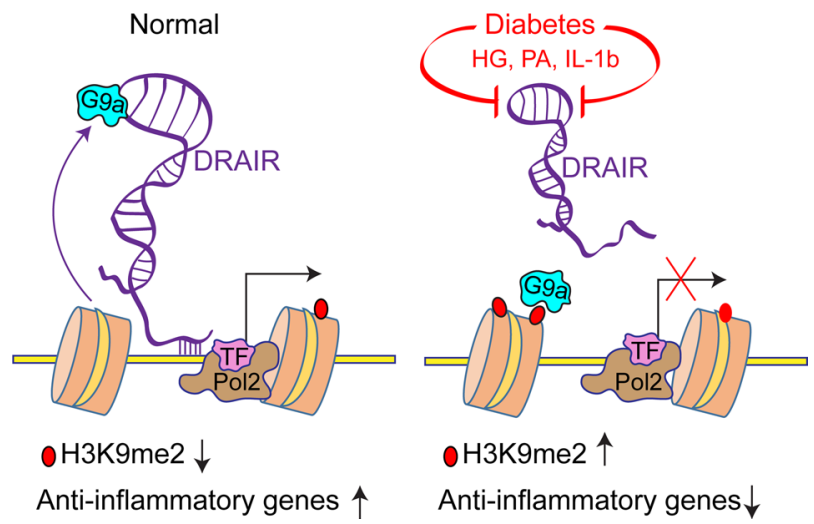

Figure 10. Schematic of DRAIR dysregulation in diabetes and epigenetic mechanisms of DRAIR actions. Under normal physiological conditions, IncRNA DRAIR reduces enrichment of repressive histone modifications, such as H3K9me2, at key antiinflammatory genes, by preventing recruitment of histone methyltransferase G9a (EHMT2), which allows the normal expression of these antiinflammatory genes. However, under diabetic conditions, downregulation of DRAIR and upregulation of C9a reverses these events, leading to repression of antiinflammatory genes, activation of monocytes, and chronic inflammation.

The key antiinflammatory gene, ILIRN, was one of the most highly downregulated genes in T2D monocytes. Its protein product IL-1Ra is a member of the IL-1 family that binds to IL-1 receptors and inhibits their responses. IL-1Ra expression is increased by proinflammatory agents and in chronic inflammatory diseases and plays an important role in host defense against inflammation (37). Experimental evidence from animal models and clinical trials using IL-1Ra (Anakinra) have underscored its role in curbing inflammation in diabetes and atherosclerosis $(38,39)$. Disruption in the balance between pathological and protective factors could promote chronic inflammation (5). Our data showing IL1RN is downregulated in T2D monocytes support the presence of such imbalances and highlight the importance of further understanding the mechanisms and factors that inhibit or repress endogenous antiinflammatory networks in T2D.

Several IncRNAs are reported to regulate the inflammatory phenotype in macrophages (16), but the role of monocyte IncRNAs in human T2D is poorly understood. In this study, we demonstrated that hundreds of IncRNAs were differentially expressed in $\mathrm{CD}_{1} 4^{+}$monocytes from subjects with $\mathrm{T} 2 \mathrm{D}$ versus controls, including multiple divergent transcripts whose functions in the monocyte/macrophage phenotype are not known. One of our key findings is that a divergent IncRNA DRAIR is downregulated in T2D and regulates antiinflammatory functions and genes, such as ILIRN and CPEB2, in monocytes and macrophages. ILIRN can be induced by several agents, including cytokines and viral and bacterial components, and it is a known antiinflammatory factor (37). CPEB members are regulated by inflammation and hypoxia $(30,40)$, but mechanisms of their regulation and action in monocytes are unclear. Our studies uncovered potentially novel lncRNA (DRAIR) dependent mechanisms in the regulation of IL1RN and CPEB2. In addition, we also demonstrated a potentially unknown antiinflammatory function of CPEB2 in monocytes. Cytoplasmic localization of CPEB2 protein suggests it may act via posttranscriptional mechanisms to regulate inflammatory genes $(30,40)$. Interestingly, we found CPEB2 knockdown also reduced DRAIR expression, suggesting that posttranscriptional regulation of $D R A I R$ by $C P E B 2$ protein may also contribute to its antiinflammatory effects.

Furthermore, we found that DRAIR regulates key macrophage genes (CD68 and CD36) and an antiapoptotic gene $M C L 1$ (41) involved in macrophage differentiation, apoptosis, and phagocytosis. Because these processes play key roles in the pathophysiological functions of macrophages associated with cardiovascular disorders like atherosclerosis $(3,6), D R A I R$ downregulation in diabetes may also accelerate inflammatory cardiovascular complications. The antiinflammatory functions of DRAIR are further supported by our mouse in vivo data showing that Drair expression is downregulated in macrophages of T2D mice and that its knockdown in nondiabetic mice enhances the macrophage inflammatory phenotype. Our experimental evidence showing DRAIR inhibition by diabetic milieu and DRAIR upregulation by mediators of alternative macrophage activation, such as antiinflammatory cytokines and TF KLF4, further support the involvement of DRAIR in antiinflammatory processes in monocyte/macrophages.

We found that DRAIR is enriched in nuclear and chromatin fractions. Such nuclear IncRNAs can promote chromatin remodeling via interactions with chromatin and chromatin modifying enzymes to regulate 
target gene expression $(16,18)$. Our ChIRP-seq data demonstrate that DRAIR interacts with chromatin at the promoter of nearby $C P E B 2$, as well as other genomic loci on multiple chromosomes. Notably, our results uncover a key DRAIR-chromatin binding site located in the OPTC gene (OPTC-Dbs) that interacts with the upstream region of $C H I T 1$ and $C H I 3 L$ genes and might play a key role in DRAIR-induced expression of $C H I T 1$ and $C H I 3 L$, genes associated with monocyte to macrophage differentiation (33). On the other hand, our ChIRP-seq analysis did not reveal DRAIR-chromatin interactions upstream of other DRAIR target genes validated in this study, such as ILIRN and MCL1. However, several genes located near Dbs $( \pm 250 \mathrm{~kb})$, including CHIT1 discussed above, were dysregulated in T2D monocytes (Supplemental Table 3). These results indicate that DRAIR regulates some genes via direct interaction with chromatin — and others possibly through different mechanisms such as interaction with chromatin-modifying proteins.

Accordingly, we found that interaction of DRAIR with the H3K9me2-methyltransferase G9a can regulate key target antiinflammatory genes. G9a can regulate gene expression via targeting transcriptionally active chromatin and interaction with 1 ncRNAs such as Kcnq1ot1 in cancer $(42,43)$. Previous studies showed dysregulation of $\mathrm{H} 3 \mathrm{~K} 9 \mathrm{me} 2$ in diabetes (35), but the role of G9a has not been explored. Interestingly, our RNA-seq data show that G9a expression is increased in T2D monocytes, and treatment of THP1 monocytes with HG and PA also increased G9a protein levels, indicating a potential role for G9a in monocyte functions in T2D. We found that DRAIR reduces promoter enrichment of G9a and the corresponding repressive mark $\mathrm{H} 3 \mathrm{~K} 9 \mathrm{me} 2$, along with upregulation of antiinflammatory genes such as $I L 1 R N$ and CPEB2. This was further supported by our observation that $E H M T 2$ (G9a) knockdown could increase the expression of antiinflammatory genes that are also regulated by $D R A I R$. Therefore, DRAIR downregulation and G9a upregulation in T2D may act cooperatively in mechanisms associated with downregulation of antiinflammatory pathways in monocytes and chronic inflammation (Figure 10). However, DRAIR may also operate via G9a-independent mechanisms - such as regulation of $\mathrm{H} 3 \mathrm{~K} 27 \mathrm{me} 3$, as in the case of FCGR3B - but further studies are needed to examine the role of $\mathrm{H} 3 \mathrm{~K} 27 \mathrm{me} 3$ in DRAIR functions.

Our study also has limitations. The T2D subjects evaluated were relatively young; hence, it is unclear if $D R A I R$ is also dysregulated in more longstanding T2D. In addition, we could not determine whether overexpression/reconstitution of Drair in $d b / d b$ mice or other mouse models of T2D can attenuate inflammation or metabolic parameters because the technology to overexpress nuclear lncRNAs in vivo is not well developed. In addition, Drair knockdown did not inhibit Il1rn expression in mice PMs, instead showing a slight but not significant increase. These results suggest either a feedback response to increase inflammation or species-specific regulation. However, most importantly, DRAIR knockdown promoted inflammatory phenotype in both mice and humans, supporting its conserved antiinflammatory functions. But further work is needed to determine if the mouse and human orthologs operate through similar molecular mechanisms. We are also aware that genetic variations affecting the expression and function of 1 ncRNAs can be associated with cardiometabolic disease $(44,45)$. Therefore, we searched DRAIR locus for genetic variants using The Cardiovascular Disease Knowledge Portal (https://cvd.hugeamp. org). However, we did not find any single nucleotide polymorphisms in the DRAIR locus that have significant association with human cardiovascular disease.

In summary, our results derived from multiple complementary approaches demonstrate that DRAIR regulates target gene expression in monocytes/macrophages via molecular and epigenetic mechanisms including direct interaction with chromatin and binding to key chromatin modifying proteins (Figure 10). Our findings emphasize the emerging role of antiinflammatory lncRNAs in metabolic diseases acting via RNA binding proteins and epigenetic mechanisms (23). Further understanding of such endogenous protective factors could aid in the development of much-needed therapies to ameliorate chronic inflammation and T2D complications.

\section{Methods}

Human CD14+ monocytes and THP1 monocytic cell line. Fasting blood $(15 \mathrm{~mL})$ was collected from T2D and control volunteers (Supplemental Table 1). PBMCs from these samples were isolated and CD $14^{+}$monocytes purified by negative selection using magnetic beads (Miltenyi Biotech) as described (21). RNA from these samples were used for RNA-seq analysis and qPCR validation at City of Hope. For some in vitro experiments, human $\mathrm{CD}_{14}{ }^{+}$monocytes from healthy volunteers were obtained from All Cells. Blood samples collected at City of Hope were used for all transfection experiments with human monocytes. The CD $14^{+}$monocytes from Ficoll-purified PBMC were isolated by immunomagnetic negative selection using EasySep Human Monocyte 
Isolation Kit (catalog 19059, Stemcell Technologies). Human THP1 monocytic cell line (American Type Culture Collection [ATCC]) was used to characterize DRAIR functions and mechanisms of actions. THP1 cells were cultured in RPMI containing 10\% FBS, penicillin/streptomycin (Pen/Strp; 100 U/100 $\mu \mathrm{g}$ per mL), $2 \mathrm{mM}$ glutamine, $5.5 \mathrm{mM}$ glucose, and $50 \mu \mathrm{M} \beta$-mercaptoethanol. CD14 ${ }^{+}$monocytes were cultured in same medium without $\beta$-mercaptoethanol. Where indicated, monocytes were treated with NG for 72 hours (5.5 $\mathrm{mM})$, HG (25 mM glucose) for 72 hours, PA $(200 \mu \mathrm{M})$ for 24 hours, and HG + PA (PA added 48 hours after culturing in HG). Cells were then lysed in QIAzol (Qiagen) for RNA extraction. Human primary monocytes were also differentiated into macrophages using M-CSF1 $(25 \mathrm{ng} / \mathrm{mL})$ for up to 1 week, while THP1 cells were differentiated using PMA (20 ng/mL, up to 48 hours) and treated as indicated.

Isolation of mice PMs. Male $d b / d b$ mice model of T2D (BKS.Cg- $\mathrm{m}^{+/+} \mathrm{epr}^{\mathrm{db}} / \mathrm{J}$, catalog 00642), nondiabetic control $d b /+$ mice, and C57BL/6 mice were obtained from the Jackson Laboratory. Thioglycolate-elicited PMs were isolated from 10- to 12-week-old $d b / d b$ and $d b /+$ mice and C57BL/6 mice as described $(21,22)$. PMs were plated in RPMI supplemented with $11 \mathrm{mM}$ glucose and Pen/Strp for 1 hour, washed $3 \times$ with PBS, and RNA extracted. Mouse macrophage cell line RAW264.7 (ATCC, TIB-71) was cultured as described (21).

$R N A$ extraction and gene expression analysis. RNA was isolated using RNeasy mini kit with on-column DNase I digestion (Qiagen). Reverse transcription followed by qPCR was performed by preparing cDNAs with Prime Script RT Master Mix (Takarabio) or QuantiTect RT-Kit (Qiagen), and qPCR with SYBR Green reagent using indicated gene primers (Supplemental Table 4) on a 7500 Fast Real-Time PCR system (Thermo Fisher Scientific). Data were analyzed using the $2^{-\triangle \Delta C t}$ method to determine relative gene expression after normalization with internal control genes (RPLPO, HPRT1, and PPIA for human) and (Rplp0 and Ppia for mouse genes) $(21,22)$.

$R N A$-seq analysis. Total RNA from CD14 $4^{+}$monocytes of T2D and control subjects ( $n=5$ each) was subjected to strand-specific RNA-seq on the Hi-Seq 2500 platform (Illumina). The data were analyzed using publicly available bioinformatics tools as described $(21,22)$. Raw sequences were aligned to the human reference genome hg19 using TopHat. RefSeq gene counts were normalized by trimmed mean of M value (TMM) method. DEGs were identified using Bioconductor package edgeR using criteria of fold change $\geq 2$, FDR $<0.05$, and average coverage $\geq 1$ in at least 1 sample. DEGs were further analyzed by DAVID Functional Annotation Tool, IPA (Qiagen), and TRAP to identify GOs, significantly enriched pathways, and enrichment of TF binding sites in the promoters, respectively. The lncRNAs lacking coding potential were identified as described earlier $(21,22)$. Enrichment of biological processes in nearby DEGs were analyzed using Enrichr web server (46). Mouse orthologous Drair was identified using liftOver tools available in UCSC browser (https://genome.ucsc.edu/cgi-bin/hgLiftOver), which converts genome coordinates and genome annotation files between assemblies. RNA-seq data are available through the NCBI's Gene Expression Omnibus (GEO) database (GSE156122; https://www.ncbi.nlm.nih.gov/geo/query/acc.cgi?acc=GSE156122).

In vitro transcription/translation assay of DRAIR. DRAIR cDNA was cloned into pcDNA3.1(+), and the resultant construct pDRAIR (Supplemental Figure $3 \mathrm{~B}$ ) was linearized by digestion with XhoI and subjected to in vitro transcription translation using the T7 TNT quick coupled transcription/translation system (Promega, catalog L1170). In parallel reactions, LUC transcript (positive control) and NTCs (negative control) were assayed. The reaction products were analyzed by Western blotting, and proteins were detected with Transcend colorimetric nonradioactive translation detection system (Promega) (22).

Transfection of plasmids and oligonucleotides. We used Dicer-substrate siRNAs (DsiRNAs) (Integrated DNA Technologies) and pooled siRNAs (Dharmacon) to knock down human DRAIR and EHMT2 (G9a), respectively, and we used LNA-modified GapmeRs (Qiagen) to target mouse Drair. THP1 monocytes, THP-1 differentiated into macrophages (PMA, $20 \mathrm{ng} / \mathrm{mL}, 24-48$ hours), human CD14 $4^{+}$monocytes differentiated into macrophages (M-CSF, $25-50 \mathrm{ng} / \mathrm{mL}$ for 7 days), and mouse RAW macrophages were transfected with indicated DsiRNs or siRNAs (20 nM) or GapmeRs (50-100 nM) using RNAiMAX (Thermo Fisher Scientific) or TransIT-KO (Mirusbio) following manufacturers' protocols. Cells were used 48-72 hours after transfection for downstream analyses.

To overexpress DRAIR, freshly isolated $\mathrm{CD} 14^{+}$monocytes were transfected with pDRAIR or control pcDNA3.1 (EV) using Human Monocyte Nucleofector Kit (Lonza) on a Nucleofector device II and program Y-001. The next day, transfected cells were treated \pm LPS $(100 \mathrm{ng} / \mathrm{mL})$ for 24 hours, and RNA were isolated. THP1 cells were also transfected with pDRAIR or control pcDNA3.1 (EV) with TransIT-2020 (Mirusbio), and stably transfected cells were selected using G418 (400 $\mu \mathrm{g} / \mathrm{mL})$. In some experiments, $D R A I R$ cDNA was cloned into lentiviral vector pLentipuro (Addgene). Lentiviruses expressing DRAIR or 
control EGFP were prepared using cotransfection with helper plasmids in HEK293T cells. THP1 cells were transduced with DRAIR and EGFP lentiviruses using polybrene (MilliporeSigma, $8 \mu \mathrm{g} / \mathrm{mL}$ ) overnight and used a week after transduction.

Mouse Drair knockdown in vivo. Male C57BL/6 mice ( 8 weeks old) were injected (i.p.) with thioglycolate (3\%), followed by i.p. injection of in vivo grade Drair Gapmer or negative control Gapmer (Qiagen) at 24 and 72 hours ( $5 \mathrm{mg} / \mathrm{kg}$ ). PMs were isolated at 96 hours for gene expression analysis.

Cloning of DRAIR promoter and transfection of reporter plasmids. Human DRAIR promoter (-1064 to +39) was PCR amplified from genomic DNA and subcloned upstream of the firefly LUC gene to generate pDRluc. Transcription factor binding sites enriched in DRAIR promoter were analyzed using TRANSFAC software (Qiagen). THP1 cells were cotransfected with pDRluc, internal control SV40-Renilla LUC, and indicated plasmids using TransIT-2020. The following day, treatments were as indicated for 24 hours, and LUC activities in cell lysates were determined using Dual-LUC Reporter Assay System in GloMax Luminometer (Promega). Firefly/Renilla ratios were reported as fold over controls.

Phagocytosis assays. THP1 cells differentiated into macrophages were transfected with siDR or siNC and treated as indicated. The next day, phagocytosis assays were performed in 96-well black plates with clear flat bottoms using FITC-labeled E. coli BioParticles as described by the manufacturer (Vybrant Phagocytosis Assay Kit, Thermo Fisher Scientific). Fluorescence from phagocytosed E.coli bioparticles was measured at 483/518 $\mathrm{nm}$ on an Infinite 200Pro plate reader (Tecan), and results were reported as arbitrary fluorescence units.

RNA isolation from nuclear, cytoplasmic, and chromatin fractions. RNA from nuclear and cytosolic fractions was purified using columns and protocols supplied with PARIS kit (Thermo Fisher Scientific). RNA fractions from chromatin and soluble nuclear extracts were isolated as described $(22,23)$. Levels of indicated transcripts levels were determined by qPCR.

RNA-FISH. RNA-FISH was performed with LNA-Cy5-labeled oligonucleotide probes targeting $D R A I R$ (EXIQON-QIAGEN) as described (22). Images were captured and processed using a Zeiss Observer Z1 wide field microscope and ZEN Blue software (Zeiss).

Western blot analysis. THP1 cells were treated as indicated with NG, HG, PA, and HP and centrifuged at $100 \mathrm{~g}$ for 10 minutes at $4^{\circ} \mathrm{C}$. Cell pellets were washed with cold PBS and lysed in Nuclear Isolation Buffer (NEB) containing $0.25 \mathrm{M}$ sucrose, $8 \mathrm{mM}$ Tris $\mathrm{HCl}$ (pH 7.4), $5 \mathrm{mM} \mathrm{MgCl}, 0.8 \%$ Triton X-100, and $1 \times$ Complete Protease inhibitor (Roche) on a rotator for 20 minutes at $4^{\circ} \mathrm{C}$. Cell lysates were centrifuged at $2500 \mathrm{~g}$ for 20 minutes at $4^{\circ} \mathrm{C}$; nuclear pellets were lysed in Laemmli sample buffer (without dye and $\beta$-mercaptoethanol) and were briefly sonicated $\left(15\right.$ seconds at $4^{\circ} \mathrm{C}$; Diagenode) to reduce viscosity. Protein concentrations were estimated using Protein Assay kit (Bio-Rad), and equal amounts of nuclear proteins were subjected to Western blotting with G9a antibody (1:1000, catalog 688851S, Cell Signaling Technology) and internal control Histone H3 antibody (1:3000, catalog ab1791, Abcam) (22). Intensities of protein bands detected by enhanced chemiluminescence were quantified using a GS-900 calibrated densitometer and Image Lab software (Bio-Rad). Results were expressed as the ratio of G9a/H3.

ChIRP. DRAIR interaction with chromatin and with nuclear proteins were determined using ChIRP assays and ChIRP-MS, respectively $(47,48)$. For ChIRP assays, THP1 cells (80 million) were fixed with glutaraldehyde for 30 minutes, washed, and lysed in ChIRP lysis buffer containing SUPERase-in RNA inhibitor (Thermo Fisher Scientific) and Complete protease inhibitors (Roche). Lysates were sonicated in Bioruptor to fragment DNA (200-1000 bp) and diluted with hybridization buffer (1:2). Then, DRAIR tiling biotinylated oligonucleotides (Stellaris) were added $(10 \mathrm{pg} / \mathrm{mL})$ and incubated overnight at $37^{\circ} \mathrm{C}$. The following day, ChIRP complexes were captured using streptavidin-magnetic beads and washed 5 times, and ChIRP-RNA and ChIRP-DNA were eluted from the beads. ChIRP-RNA was used to estimate DRAIR recovery. ChIRP-DNA was used for DNA-seq on Illumina platform (HiSeq 2500). RAW reads were aligned to the human hg19 reference genome, and Dbs on chromatin identified as described (47). ChIRP-seq data are available through the NCBI's GEO database (GSE156122). ChIRP-DNA was also analyzed by ChIRP-qPCR to validate Dbs with specific primers.

To identify DRAIR-interacting proteins, THP1 cells stably overexpressing DRAIR (400 million cells) were fixed with formaldehyde (3\%) for 30 minutes (48), and cell lysates were processed as described for ChIRP. Biotinylated oligonucleotides targeting LUC transcript were used as negative controls. Nucleic acid-protein complexes were captured using streptavidin-magnetic beads and boiled in SDS-Laemmli protein sample buffer, and eluted proteins were fractionated on precast SDS-PAGE (4\%-15\%) gels (Bio-Rad). Gels were stained with SimplyBlue SafeStain, and different regions from each lane (DRAIR and LUC probes) were subjected 
to MS at City of Hope's Mass Spectrometry Core as described $(22,23)$. Differentially interacting proteins with $D R A I R$ versus LUC probes were analyzed using Scaffold 3.0. DRAIR-interacting proteins were further analyzed by STRING database to identify enrichment of biological processes (34) and were validated using RNA pulldown and RIP assays.

RIP assays. THP1 cells were fixed with formaldehyde (1\%) for 10 minutes at room temperature, washed with ice cold PBS, lysed in nuclear isolation buffer supplemented with RNase and protease inhibitors, and centrifuged at $2500 \mathrm{~g}$ for 20 minutes at $4^{\circ} \mathrm{C}$ to collect the nuclear pellet. Nuclei were lysed in RIPA buffer, sonicated, and immunoprecipitated overnight at $4^{\circ} \mathrm{C}$ with G9a antibody (5 $\mu$ g, catalog $\left.68851 \mathrm{~S}\right)$, SUV39H1 antibody (5 $\mu \mathrm{g}$, catalog 8729S), or negative control IgG (catalog 2729S) (all from Cell Signaling Technology). Immune complexes were collected on IgG-magnetic beads, washed 5 times with RIPA buffer, and incubated in elution buffer containing proteinase $\mathrm{K}$ at $55^{\circ} \mathrm{C}$ for 30 minutes. RNA from supernatants was purified using RNeasy MinElute Cleanup Kit (Qiagen) and analyzed by qPCR.

RNA pulldown assays. The pcDNA3.1(+) pDRAIR and pcDNA3.1(-) expressing DRAIR anti-sense (pDRAIR-AS, Supplemental Figure 3C) were linearized to prepare biotinylated DRAIR and DRAIR-AS probes, respectively, by in vitro transcription with T7-RNA polymerase using Biotin RNA Labeling Mix kit (Roche). Nuclear extracts from THP1 cells (1 mg protein) were incubated with $1 \mu \mathrm{g}$ of biotinylated probes, and RNA pulldown assays were performed as described (22). Proteins eluted from biotinylated RNA-protein complexes were subjected to immunoblotting with G9a antibody (1:1000). Protein bands were detected using Enhanced Chemiluminescence kit (Perkin Elmer).

ChIP. ChIP assays were performed as described previously $(22,49)$, with some modifications. THP1 cells crosslinked with $1 \%$ formaldehyde were lysed in nuclear isolation buffer $\left(20\right.$ minutes, $\left.4^{\circ} \mathrm{C}\right)$ and centrifuged $\left(2500 g, 20\right.$ minutes) at $4^{\circ} \mathrm{C}$. Nuclear pellets were lysed in ChIP lysis buffer, and chromatin was sheared by sonication using a Bioruptor. Sonicated nuclear lysates were diluted 1:10, and lysates containing equal amounts of DNA were immunoprecipitated using antibodies against H3K9me2 (catalog 4658S), G9a (catalog 68851S), or H3K27me3 (catalog 9733S) obtained from Cell Signaling Technology, or KLF4 (10 $\mu$ g, catalog AF3640, R\&D Systems). Immunocomplexes were captured using IgG magnetic beads, washed, and eluted as indicated $(22,49)$, except that high salt wash was performed 2 times. ChIP DNA was reverse crosslinked overnight at $67^{\circ} \mathrm{C}$ and treated with RNAse A $(10 \mu \mathrm{g} / \mathrm{mL})$ for 30 minutes at $37^{\circ} \mathrm{C}$. DNA was extracted using QIAquick PCR Purification Kit. ChIP-DNA was analyzed in triplicate by ChIP-qPCR using indicated primers and SYBR Green reagent on the 7500 Fast Real-Time PCR system. The ChIP-qPCR data were analyzed using

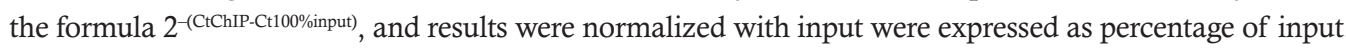

THP1 monocyte-EC binding assays. THP1 monocytes transfected with siDR or siNC were treated with or without TNF- $\alpha$ for 5 hours ( $10 \mathrm{ng} / \mathrm{mL}$ ), labeled with DAPI ( $5 \mathrm{ng} / \mathrm{mL}, 20$ minutes), and washed twice with PBS. Then, labeled monocytes $\left(125,000\right.$ per $\left.\mathrm{cm}^{2}\right)$ were incubated with confluent HUVECs in 24-well plates in serum-depletion medium (MCDB-131 [MilliporeSigma] containing 2.5\% FBS and $1 \times$ antibiotic/antimycotic agents) for 30 minutes at $37^{\circ} \mathrm{C}$. Nonadherent monocytes were removed by washing twice with PBS. EC-bound monocytes were fixed with $1 \%$ paraformaldehyde for 15 minutes and washed, and images from multiple wells/group were captured using a fluorescence microscope $(\times 100$ magnification). Bound monocytes were counted with ImageJ software (NIH), and results are expressed as monocytes/field (mean \pm SD).

Statistics. Graphpad Prism (7.0 and above) software was used to perform statistical analysis. Data are represented as mean \pm SD of experiments performed at least in triplicate. Shapiro-Wilk normality test was used to test normal distribution of each sample group. Comparisons between 2 groups were performed using 2-tailed unpaired $t$ tests. Comparison among more than 2 groups were performed using 1- or 2-way ANOVA, followed by multiple-comparison tests as indicated in the figure legends. $P<0.05$ was considered statistically significant.

Study approval. All human blood samples were collected after written informed consent from T2D and control volunteers. The study was approved by the IRBs at City of Hope and Baylor College of Medicine. Mouse studies were approved by IACUC at City of Hope and conducted in accordance with the Guide for the Care and Use of Laboratory Animals (National Academies Press, 2011).

\section{Author contributions}

MAR conceptualized the work, designed and performed most experiments, and wrote the manuscript. VA, RG, MW, LL, LZ, and MA performed experiments and analyzed data. XW, ZC, and VST performed bioinformatics analysis. S. Das performed experiments, assisted with experimental design, and 
edited the manuscript. S. Devaraj provided human monocytes for RNA-seq analysis. RN conceptualized the work, edited the manuscript, acquired funding, and supervised the study. RN and MAR are the guarantors of this work and, as such, had full access to all the data in the study and take responsibility for the integrity of the data and the accuracy of the data analysis.

\section{Acknowledgments}

We are grateful to the Integrative Genomics Core (City of Hope) for assistance with analysis of RNA-seq and ChIRP-seq data, and the Proteomics Core (City of Hope) for mass spectroscopy analysis of ChIRP samples. We thank Harry Scott and Kaustabh Ghosh (University of California - Riverside, Riverside California, USA) for assistance with endothelial cell and monocyte binding studies. Research reported in this publication included work performed in the Integrative Genomics, DNA/RNA Synthesis, and Mass Spectrometry and Proteomics Cores (supported by the National Cancer Institute of the NIH under grant no. P30CA033572), and the Light Microscopy Core of City of Hope. The content is solely the responsibility of the authors and does not necessarily represent the official views of the NIH. This study was supported by grants from the NIH: R01 DK065073, R01 HL106089, and R01 DK081705 (to RN) and a pilot grant from the Arthur Riggs Diabetes and Metabolism Research Institute of City of Hope (to MAR). VA was supported by a Predoctoral fellowship grant, and RG was supported by a postdoctoral fellowship grant, both from the American Heart Association.

Address correspondence to: Rama Natarajan or Marpadga A. Reddy, Department of Diabetes Complications and Metabolism, Arthur Riggs Diabetes and Metabolism Research Institute and Beckman Research Institute of City of Hope, 1500 East Duarte Road, Duarte, California 91010, USA. Phone: 626.218.2289; Email: rnatarajan@coh.org (RN). Phone: 626.218.3671; Email: mreddy@coh.org (MAR).

1. Bhupathiraju SN, Hu FB. Epidemiology of obesity and diabetes and their cardiovascular complications. Circ Res. 2016;118(11):1723-1735.

2. Donath MY. Targeting inflammation in the treatment of type 2 diabetes: time to start. Nat Rev Drug Discov. 2014;13(6):465-476.

3. Murray PJ, Wynn TA. Protective and pathogenic functions of macrophage subsets. Nat Rev Immunol. 2011;11(11):723-737.

4. Serhan CN, Levy BD. Resolvins in inflammation: emergence of the pro-resolving superfamily of mediators. J Clin Invest. 2018;128(7):2657-2669.

5. Rask-Madsen C, King GL. Vascular complications of diabetes: mechanisms of injury and protective factors. Cell Metab. 2013;17(1):20-33

6. Kanter JE, et al. Monocytes and macrophages as protagonists in vascular complications of diabetes. Front Cardiovasc Med. 2020;7:10.

7. Forbes JM, Cooper ME. Mechanisms of diabetic complications. Physiol Rev. 2013;93(1):137-188.

8. Nagareddy PR, et al. Hyperglycemia promotes myelopoiesis and impairs the resolution of atherosclerosis. Cell Metab. 2013;17(5):695-708.

9. Lamharzi N, et al. Hyperlipidemia in concert with hyperglycemia stimulates the proliferation of macrophages in atherosclerotic lesions: potential role of glucose-oxidized LDL. Diabetes. 2004;53(12):3217-3225.

10. Esposito K, et al. Inflammatory cytokine concentrations are acutely increased by hyperglycemia in humans: role of oxidative stress. Circulation. 2002;106(16):2067-2072.

11. Reddy MA, et al. Epigenetic mechanisms in diabetic complications and metabolic memory. Diabetologia. 2015;58(3):443-455.

12. Malozowski S, Sahlroot JT. Interleukin-1-receptor antagonist in type 2 diabetes mellitus. N Engl J Med. 2007;357(3):302-303.

13. Donath MY. Multiple benefits of targeting inflammation in the treatment of type 2 diabetes. Diabetologia. 2016;59(4):679-682

14. Back M, Hansson GK. Anti-inflammatory therapies for atherosclerosis. Nat Rev Cardiol. 2015;12(4):199-211.

15. Ridker PM, et al. Reduction in C-reactive protein and LDL cholesterol and cardiovascular event rates after initiation of rosuvastatin: a prospective study of the JUPITER trial. Lancet. 2009;373(9670):1175-1182.

16. Chen YG, et al. Gene regulation in the immune system by long noncoding RNAs. Nat Immunol. 2017;18(9):962-972.

17. Fitzgerald KA, Caffrey DR. Long noncoding RNAs in innate and adaptive immunity. Curr Opin Immunol. 2014;26:140-146.

18. Leung A, et al. Linking diabetic vascular complications with LncRNAs. Vascul Pharmacol. 2019;114:139-144.

19. Simion V, et al. LncRNAs in vascular biology and disease. Vascul Pharmacol. 2019;114:145-156.

20. Das S, et al. Role of epigenetic mechanisms regulated by enhancers and long noncoding RNAs in cardiovascular disease. Curr Opin Cardiol. 2020;35(3):234-241.

21. Reddy MA, et al. Regulation of inflammatory phenotype in macrophages by a diabetes-induced long noncoding RNA. Diabetes. 2014;63(12):4249-4261.

22. Das S, et al. Diabetes mellitus-induced long noncoding RNA Dnm3os regulates macrophage functions and inflammation via nuclear mechanisms. Arterioscler Thromb Vasc Biol. 2018;38(8):1806-1820.

23. Stapleton K, et al. Novel long noncoding RNA, macrophage inflammation-suppressing transcript (MIST), regulates macrophage activation during obesity. Arterioscler Thromb Vasc Biol. 2020;40(4):914-928.

24. Zhang H, et al. Deep RNA sequencing uncovers a repertoire of human macrophage long intergenic noncoding RNAs modulated by macrophage activation and associated with cardiometabolic diseases. J Am Heart Assoc. 2017;6(11):e007431.

25. Devaraj S, et al. Diabetes is a proinflammatory state: a translational perspective. Expert Rev Endocrinol Metab. 2010;5(1):19-28.

26. Shanmugam N, et al. High glucose-induced expression of proinflammatory cytokine and chemokine genes in monocytic cells. 
Diabetes. 2003;52(5):1256-1264.

27. Yun JM, et al. Epigenetic regulation of high glucose-induced proinflammatory cytokine production in monocytes by curcumin. J Nutr Biochem. 2011;22(5):450-458.

28. Murray PJ. Macrophage polarization. Annu Rev Physiol. 2017;79:541-566.

29. Luo S, et al. Divergent lncRNAs regulate gene expression and lineage differentiation in pluripotent cells. Cell Stem Cell. 2016;18(5):637-652

30. Ivshina M, et al. CPEB regulation of TAK1 synthesis mediates cytokine production and the inflammatory immune response. Mol Cell Biol. 2015;35(3):610-618.

31. Liao X, et al. Krüppel-like factor 4 regulates macrophage polarization. J Clin Invest. 2011;121(7):2736-2749.

32. Schofield EC, et al. CHiCP: a web-based tool for the integrative and interactive visualization of promoter capture Hi-C datasets. Bioinformatics. 2016;32(16):2511-2513.

33. Di Rosa M, et al. Evaluation of CHI3L-1 and CHIT-1 expression in differentiated and polarized macrophages. Inflammation. 2013;36(2):482-492.

34. Szklarczyk D, et al. STRING v11: protein-protein association networks with increased coverage, supporting functional discovery in genome-wide experimental datasets. Nucleic Acids Res. 2019;47(D1):D607-D613.

35. Miao F, et al. Genome-wide analysis of histone lysine methylation variations caused by diabetic conditions in human monocytes. J Biol Chem. 2007;282(18):13854-13863.

36. Chittock EC, et al. Molecular architecture of polycomb repressive complexes. Biochem Soc Trans. 2017;45(1):193-205.

37. Arend WP, et al. Interleukin-1 receptor antagonist: role in biology. Annu Rev Immunol. 1998;16:27-55.

38. Larsen CM, et al. Interleukin-1-receptor antagonist in type 2 diabetes mellitus. N Engl J Med. 2007;356(15):1517-1526

39. Libby P. Interleukin-1 beta as a target for atherosclerosis therapy: biological basis of CANTOS and beyond. J Am Coll Cardiol. 2017;70(18):2278-2289

40. Chen PJ, Huang YS. CPEB2-eEF2 interaction impedes HIF-1 $\alpha$ RNA translation. EMBO J. 2012;31(4):959-971.

41. Daigneault M, et al. The identification of markers of macrophage differentiation in PMA-stimulated THP-1 cells and monocyte-derived macrophages. PLoS One. 2010;5(1):e8668.

42. Long Y, et al. How do lncRNAs regulate transcription? Sci Adv. 2017;3(9):eaao2110.

43. Cao H, et al. Recent progress in histone methyltransferase (G9a) inhibitors as anticancer agents. Eur J Med Chem. 2019;179:537-546.

44. Giral H, et al. Into the wild: GWAS exploration of non-coding RNAs. Front Cardiovasc Med. 2018;5:181.

45. Foulkes AS, et al. Nonconserved long intergenic noncoding RNAs associate with complex cardiometabolic disease traits. Arterioscler Thromb Vasc Biol. 2021;41(1):501-511.

46. Kuleshov MV, et al. Enrichr: a comprehensive gene set enrichment analysis web server 2016 update. Nucleic Acids Res. 2016;44(W1):W90-W97.

47. Chu C, et al. Genomic maps of long noncoding RNA occupancy reveal principles of RNA-chromatin interactions. Mol Cell. 2011;44(4):667-678

48. Chu C, et al. Systematic discovery of Xist RNA binding proteins. Cell. 2015;161(2):404-416.

49. Das S, et al. Regulation of angiotensin II actions by enhancers and super-enhancers in vascular smooth muscle cells. Nat Commun. 2017;8(1):1467. 